\title{
The Riemann problem for a class of resonant hyperbolic systems of balance laws
}

\section{Le problème de Riemann pour une classe de systèmes hyperboliques non-conservatifs avec résonance}

\author{
Paola Goatin ${ }^{\text {a }}$, Philippe G. LeFloch ${ }^{\text {b,* }}$ \\ ${ }^{a}$ Laboratoire de mathématiques, U.F.R. des sciences et techniques, université de Toulon et du Var, avenue de l'université, \\ 83957 La Garde Cedex, France \\ ${ }^{\mathrm{b}}$ Centre de mathématiques appliquées \& Centre national de la recherche scientifique, U.M.R. 7641, École polytechnique, \\ 91128 Palaiseau Cedex, France
}

Received 20 February 2003; accepted 27 February 2004

Available online 29 July 2004

\begin{abstract}
We solve the Riemann problem for a class of resonant hyperbolic systems of balance laws. The systems are not strictly hyperbolic and the solutions take their values in a neighborhood of a state where two characteristic speeds coincide. Our construction generalizes the ones given earlier by Isaacson and Temple for scalar equations and for conservative systems. The class of systems under consideration here includes, in particular, a model from continuum physics that describes the evolution of a fluid flow in a nozzle with discontinuous cross-section.

(C) 2004 L'Association Publications de l'Institut Henri Poincaré. Published by Elsevier B.V. All rights reserved
\end{abstract}

\section{Résumé}

Nous résolvons le problème de Riemann pour une classe de systèmes hyperboliques non-conservatifs et résonants. Ces systèmes ne sont pas strictement hyperboliques et les solutions considérées prennent leurs valeurs au voisinage d'un état constant où deux des vitesses caractéristiques coincident. Notre construction généralise celle donnée précédemment par Isaacson et Temple pour les équations scalaires et les systèmes conservatifs. La classe générale de systèmes étudiée ici comprend, en particulier, un modèle important de la dynamique des milieux continus qui décrit l'évolution d'un fluide dans une tuyère dont la section est discontinue.

C 2004 L'Association Publications de l'Institut Henri Poincaré. Published by Elsevier B.V. All rights reserved

\footnotetext{
* Corresponding author.

E-mail addresses: goatin@cmap.polytechnique.fr (P. Goatin), lefloch@cmap.polytechnique.fr (P.G. LeFloch).
} 
Keywords: Nozzle; Fluid flow; Hyperbolic conservation law; Nonconservatif; Balance law; Resonant; Entropy solution; Shock wave; Riemann problem

\section{Introduction}

In this paper we study the Riemann problem for a general class of nonlinear hyperbolic systems of balance laws, which is motivated by the Euler system of compressible fluids in a nozzle with variable cross-section (see (1.9) below). Precisely, we are interested in the Riemann problem for the nonlinear hyperbolic system

$$
\begin{aligned}
& \partial_{t} u+\partial_{x} f(u, a)=g(u, a) \partial_{x} a, \\
& \partial_{t} a=0,
\end{aligned}
$$

with initial data consisting, by definition, of two constant states $\left(a_{L}, u_{L}\right)$ and $\left(a_{R}, u_{R}\right)$ :

$$
(u, a)(x, 0)= \begin{cases}\left(u_{L}, a_{L}\right), & x<0 \\ \left(u_{R}, a_{R}\right), & x>0 .\end{cases}
$$

The unknowns are the two functions $u=u(x, t) \in \mathbb{R}^{n}$ and $a=a(x) \in \mathbb{R}$.

It is assumed that the flux-function $f=f(u, a)$ in (1.1) is a given smooth mapping such that, for each value $a \in \mathbb{R}, f(\cdot, a): \mathbb{R}^{n} \mapsto \mathbb{R}^{n}$ is strictly hyperbolic; that is, for each $u \in \mathbb{R}^{n}$ the Jacobian matrix $D_{u} f(u, a)$ admits $n$ real and distinct eigenvalues

$$
\lambda_{1}(u, a)<\cdots<\lambda_{n}(u, a)
$$

and, therefore, corresponding basis of left- and right-eigenvectors $l_{i}(u, a), r_{i}(u, a)(i=1, \ldots, n)$, normalized so that

$$
\left|r_{i}(u, a)\right|=1, \quad l_{i}(u, a) \cdot r_{i}(u, a)=1, \quad l_{i}(u, a) \cdot r_{j}(u, a)=0 \quad \text { if } i \neq j .
$$

We also assume that each characteristic field of $D_{u} f(u, a)$ is either genuinely nonlinear or linearly degenerate, that is, for each $i=1, \ldots, n$, the function $(u, a) \mapsto \nabla_{u} \lambda_{i}(u, a) \cdot r_{i}(u, a)$ never vanishes or vanishes identically, respectively.

In addition, we observe that Eq. (1.2) trivially corresponds to a linearly degenerate field with eigenvalue

$$
\lambda_{0}:=0 \text {. }
$$

We are interested in studying the problem (1.1)-(1.3) when the Riemann data lie in a neighborhood of a state $\left(u_{*}, a_{*}\right)$ at which one of the wave speeds of (1.1) also vanishes, that is, we assume that for some index $k$

$$
\lambda_{k}\left(u_{*}, a_{*}\right)=\lambda_{0} .
$$

The $k$-characteristic field is assumed to be genuinely nonlinear, so after normalization

$$
\left(\nabla_{u} \lambda_{k} \cdot r_{k}\right)\left(u_{*}, a_{*}\right)>0 .
$$

Throughout this paper, we restrict attention to data in the ball $\mathcal{B}\left(u_{*}, \delta_{0}\right)$ with center $u_{*}$ and (small) radius $\delta_{0}>0$ and we impose on the functions $f$ and $g$ the following two conditions

$$
\begin{aligned}
& \left(l_{k} \cdot\left(\partial_{a} f-g\right)\right)\left(u_{*}, a_{*}\right) \neq 0 \quad \text { (we assume that it is negative), } \\
& \left(l_{k} \cdot D_{u} g \cdot r_{k}\right)\left(u_{*}, a_{*}\right) \neq 0 \quad \text { (we assume that it is negative) }
\end{aligned}
$$

which, as we will show, give the generic structure of elementary waves near $\left(u_{*}, a_{*}\right)$. By continuity, we can always assume that (1.6)-(1.7) still hold for all $u \in \mathcal{B}\left(u_{*}, \delta_{0}\right)$ and $a \in \mathcal{B}\left(a_{*}, \delta_{0}\right)$. 
Hyperbolic systems of balance laws, having the form of conservation laws with a source arise in many applications. Most notably, the Euler equations for a fluid flow in a nozzle with cross-sectional area $a=a(x)$ read (see for instance [11]):

$$
\begin{aligned}
& \partial_{t} \rho+\partial_{x}(\rho u)=-\frac{\rho u}{a} \partial_{x} a, \\
& \partial_{t}(\rho u)+\partial_{x}\left(\rho u^{2}+p\right)=-\frac{\rho u^{2}}{a} \partial_{x} a, \\
& \partial_{t}(\rho e)+\partial_{x}((\rho e+p) u)=-\frac{(\rho e+p) u}{a} \partial_{x} a .
\end{aligned}
$$

Here, $\rho>0$ denotes the density of the fluid, $u$ the velocity, $p$ the pressure, and $e$ is the total (internal and kinetic) energy. The equations express balance laws for the mass, momentum, and total energy of the fluid through the nozzle.

We emphasize that the model (1.1)-(1.2) has two important features. On one hand, it contains a nonconservative product $g(u, a) \partial_{x} a$ which cannot make sense within the framework of the theory of distributions. Instead, a rigorous definition of weak solutions must be based on the theory of nonconservative products due to Dal Maso, LeFloch, and Murat [22-24,12]. See in particular LeFloch [23] for a review of several applications, including the model (1.9) above. On the other hand, (1.1)-(1.2) is non-strictly hyperbolic: for works on resonant systems we refer to Marchesin and Paes-Leme [31], Embid, Goodman, and Majda [13], Glimm, Marschall, and Plohr [14], Isaacson and Temple [18,19], Liu [28-30], Lien [27], and Marchesin, Plohr, and Schecter [32]. The construction proposed in this paper can be regarded as an extension to resonant nonconservative systems of [18] (conservative systems) and [19] (scalar, nonconservative equations). For other important related works, including the construction of Riemann solutions and numerical schemes for equations similar to (1.9), we refer to Amadori, Gosse, and Guerra [1,2,15], Andrianov and Warnecke [3-5], Asakura [6], Botchorishvili, Perthame, and Vasseur [7], Bouchut [8], Chen and Glimm [9], Greenberg and Leroux [16], Jin and Wen [20,21], and LeFloch and Thanh [26].

\section{Preliminaries}

By setting

$$
U:=(u, a), \quad F(U):=(f(u, a), 0), \quad G(U):=\left[\begin{array}{cc}
0 & g(u, a) \\
0 & 0
\end{array}\right],
$$

the set of Eqs. (1.1)-(1.2) can be regarded as a nonconservative system of $n+1$ equations:

$$
U_{t}+\left(D_{U} F(U)-G(U)\right) U_{x}=0 .
$$

We denote by $R_{i}(U), i=0, \ldots, n$, the corresponding right-eigenvectors of the matrix $D_{U} F(U)-G(U)$. Clearly, we have $R_{i}=\left(r_{i}, 0\right), i=1, \ldots, n$. The vector $R_{0}$ is associated with the eigenvalue $\lambda_{0}$ and will be determined shortly.

First, we discuss some consequences of our assumptions (1.5)-(1.8). By the implicit function theorem, (1.6) guarantees that the equation $\lambda_{k}(u, a)=0$ defines (locally) a smooth $n$-dimensional manifold of $\mathbb{R}^{n+1}$,

$$
\mathcal{T}:=\left\{(u, a), u \in \mathcal{B}\left(u_{*}, \delta_{0}\right), a \in \mathcal{B}\left(a_{*}, \delta_{0}\right) / \lambda_{k}(u, a)=0\right\} \subset \mathbb{R}^{n+1},
$$

which passes through the state $U_{*}:=\left(u_{*}, a_{*}\right)$ and will be called the transition manifold. The integral curves of $r_{k}$ are transversal to $\mathcal{T}$, and this property allows us to distinguish two "half-spaces"

$$
\mathcal{T}^{ \pm}:=\left\{U \in \mathcal{B}\left(U_{*}, \delta_{0}\right) / \text { for all } V \in \mathcal{T}, R_{k}(V) \cdot(U-V) \gtrless 0\right\} .
$$

We now determine the right-eigenvector $R_{0}(u, a)$. Thanks to (1.7) the Jacobian matrix

$$
D_{U} F(U)-G(U)=\left[\begin{array}{cc}
D_{u} f & \partial_{a} f-g \\
0 & 0
\end{array}\right]
$$


has rank $n$ on $\mathcal{T}$ in a neighborhood of $U_{*}$. We claim that the eigenvectors $R_{0}$ and $R_{k}$ can be chosen outside the manifold $\mathcal{T}$ in such a way that they remain continuous across the manifold $\mathcal{T}$. This is clear for the vector field $R_{k}$. The vector $R_{0}=:\left(r_{0}, b_{0}\right)$ must satisfy

$$
\left(\partial_{a} f-g\right) b_{0}+D_{u} f \cdot r_{0}=0 .
$$

If we search for the component $r_{0}$ in the general form $r_{0}=\sum_{i=1}^{n} \alpha_{i} r_{i}$ and we multiply (2.2) by each left-eigenvector $l_{i}$ we obtain

$$
l_{i} \cdot\left(\partial_{a} f-g\right) b_{0}+\lambda_{i} \alpha_{i}=0,
$$

which determines the coefficient $\alpha_{i}$. Hence, imposing $R_{0}$ to be a unit vector, we find

$$
R_{0}=c\left(-\sum_{i=1}^{n} \frac{\lambda_{k}}{\lambda_{i}} l_{i} \cdot\left(\partial_{a} f-g\right) r_{i}, \lambda_{k}\right), \quad(u, a) \notin \mathcal{T},
$$

where the normalization coefficient $c>0$ is given by

$$
\frac{1}{c^{2}}:=\lambda_{k}^{2}+\sum_{i=1}^{n}\left(\frac{\lambda_{k}}{\lambda_{i}} l_{i} \cdot\left(\partial_{a} f-g\right)\right)^{2} .
$$

It is now easy to check that, as the state $(u, a)$ approaches the manifold $\mathcal{T}$, we have $r_{0} \rightarrow r_{k}$ and $b_{0} \rightarrow 0$, and that $R_{0}$ can be extended smoothly to the manifold and coincides with $R_{k}=\left(r_{k}, 0\right)$ on $\mathcal{T}$, in other words,

$$
R_{0}(u, a)-R_{k}(u, a) \rightarrow 0 \quad \text { as }(u, a) \text { approaches } \mathcal{T} .
$$

In particular, provided $\delta_{0}>0$ is sufficiently small, $\left|R_{0}(U)-R_{k}(U)\right| \leqslant \varepsilon$ for $\left|U-U_{*}\right| \leqslant \delta<\delta_{0}$.

To parametrize the wave curves it will be convenient to introduce at this stage a globally defined parameter $\mu_{i}(u) \in \mathbb{R}$ which should depend smoothly upon $u$ and be strictly monotone along the wave curve. More precisely, we assume that the parameter $\mu_{i}$ is given such that

$$
\nabla \mu_{i}(u) \cdot r_{i}(u) \neq 0, \quad u \in \mathcal{B}\left(u_{*}, \delta_{0}\right)
$$

(here and in the sequel $a$ is kept fixed and we will neglect it while it does not play a role). In view of condition (2.5), when the field is genuinely nonlinear a natural choice for $\mu_{i}$ is the wave speed $\lambda_{i}$, while there is no canonical choice for linearly degenerate fields. In particular, for $i=k$ we will set

$$
\mu_{k}(u)=\lambda_{k}(u), \quad u \in \mathcal{B}\left(u_{*}, \delta_{0}\right),
$$

and we will reparametrize the wave curves accordingly. For some $\delta_{1}<\delta_{0}, u_{L} \in \mathcal{B}\left(u_{*}, \delta_{1}\right)$, and $\varepsilon>0$, we will denote by $m \mapsto v_{k}\left(m ; u_{L}\right)$ the Hugoniot curve (or shock curve) consisting of all right states $u_{R}$ that can be connected to $u_{L}$ by a $k$-shock of speed $\bar{\lambda}_{k}\left(m ; u_{L}\right)$, and by $m \mapsto w_{k}\left(m ; u_{L}\right)$ the rarefaction curve, parametrized so that we can refer to

$$
m \mapsto \psi_{k}\left(m ; u_{L}\right):= \begin{cases}v_{k}\left(m ; u_{L}\right), & m \in\left(-\varepsilon, \mu_{k}\left(u_{L}\right)\right], \\ w_{k}\left(m ; u_{L}\right), & m \in\left[\mu_{k}\left(u_{L}\right), \varepsilon\right)\end{cases}
$$

as the $k$-wave curve issuing from $u_{L}$ (for details, see for example [25, Chapter VI]). We will also use the notations

$$
\begin{aligned}
& \mathcal{S}_{k}\left(u_{L}\right):=\left\{v_{k}\left(m ; u_{L}\right), m \in\left(-\varepsilon, \mu_{k}\left(u_{L}\right)\right]\right\}, \\
& \mathcal{R}_{k}\left(u_{L}\right):=\left\{w_{k}\left(m ; u_{L}\right), m \in\left[\mu_{k}\left(u_{L}\right), \varepsilon\right)\right\}, \\
& \mathcal{W}_{k}\left(u_{L}\right):=\mathcal{S}_{k}\left(u_{L}\right) \cup \mathcal{R}_{k}\left(u_{L}\right)=\left\{\psi_{k}\left(m ; u_{L}\right), m \in(-\varepsilon, \varepsilon)\right\} .
\end{aligned}
$$

Thanks to (2.5) and (1.6), we can choose the parameter $m$ to coincide with $\mu_{k}$, that is:

$$
\mu_{k}\left(\psi_{k}\left(m ; u_{L}\right)\right)=m \text {. }
$$


In this situation, by setting $\tilde{m}:=m-\mu_{k}\left(u_{L}\right)$, we have the following expansion for the shock curve

$$
v_{k}\left(m ; u_{L}\right)=u_{L}+\frac{\tilde{m}}{a\left(u_{L}\right)} r_{k}\left(u_{L}\right)+\frac{\tilde{m}^{2}}{2 a\left(u_{L}\right)^{2}}\left(D r_{k} \cdot r_{k}+b r_{k}\right)\left(u_{L}\right)+\mathcal{O}\left(\tilde{m}^{3}\right),
$$

and the corresponding shock speed satisfies

$$
\begin{aligned}
\bar{\lambda}_{k}\left(m ; u_{L}\right)= & \lambda_{k}\left(u_{L}\right)+\frac{\tilde{m}}{2 a\left(u_{L}\right)}\left(\nabla \lambda_{k} \cdot r_{k}\right)\left(u_{L}\right) \\
& +\frac{\tilde{m}^{2}}{6 a\left(u_{L}\right)^{2}}\left(\nabla\left(\nabla \lambda_{k} \cdot r_{k}\right) \cdot r_{k}+c \nabla \lambda_{k} \cdot r_{k}\right)\left(u_{L}\right)+\mathcal{O}\left(\tilde{m}^{3}\right),
\end{aligned}
$$

where $a=\nabla \mu_{k} \cdot r_{k}=\nabla \lambda_{k} \cdot r_{k} \neq 0$ and $b, c$ are smooth and real-valued functions of $u_{L}$, while the expansion of the rarefaction curve $w_{k}\left(m ; u_{L}\right)$ takes the same form as (2.9). Derivation of (2.10) gives the following expansions for the partial derivatives of $\bar{\lambda}_{k}\left(m ; u_{L}\right)$ :

$$
\begin{aligned}
& \partial_{m} \bar{\lambda}_{k}\left(m ; u_{L}\right)=\frac{1}{2}+\frac{\tilde{m}}{3 a^{2}}\left(\nabla\left(\nabla \lambda_{k} \cdot r_{k}\right) \cdot r_{k}+c \nabla \lambda_{k} \cdot r_{k}\right)+\mathcal{O}\left(\tilde{m}^{2}\right), \\
& \nabla \bar{\lambda}_{k}\left(m ; u_{L}\right) \cdot r_{k}=\frac{1}{2} \nabla \lambda_{k} \cdot r_{k}-\frac{\tilde{m}}{3 a}\left(\nabla\left(\nabla \lambda_{k} \cdot r_{k}\right) \cdot r_{k}+c \nabla \lambda_{k} \cdot r_{k}\right)+\mathcal{O}\left(\tilde{m}^{2}\right)
\end{aligned}
$$

(where $r_{k}=r_{k}\left(u_{L}\right), \lambda_{k}=\lambda_{k}\left(u_{L}\right), a=a\left(u_{L}\right)=\nabla \lambda_{k} \cdot r_{k}\left(u_{L}\right)$ ).

Given some state $\left(u_{-}, a_{-}\right)$we now investigate the properties of two important curves, that will play a central role in the construction of the solution of the Riemann problem. First we study the standing wave curve, denoted by $m \mapsto(\varphi, \alpha)\left(m ; u_{-}, a_{-}\right)$, made of all states which can be attained by using time-independent smooth solution of (1.1)-(1.2). Second, we consider the composite transformed standing curve, made of states which can be reached by a standing wave followed by a shock wave with zero speed. We state the properties of these curves in the following two lemmas.

Lemma 2.1. Given some state $\left(u_{-}, a_{-}\right) \in \mathcal{T}$ consider the standing wave solution of (1.1)-(1.2), denoted by $m \mapsto$ $(\varphi, \alpha)\left(m ; u_{-}, a_{-}\right)$and determined by

$$
\begin{aligned}
& (\varphi, \alpha)^{\prime}=\gamma(m) R_{0}(\varphi, \alpha), \\
& \varphi(0)=u_{-}, \quad \alpha(0)=a_{-},
\end{aligned}
$$

for some smooth scalar function $\gamma(m)$ bounded away from zero and such that $\gamma(0)=1 / a\left(u_{-}\right)$. Then we have

$$
\alpha^{\prime}(0)=0 \quad \text { and } \quad \alpha^{\prime \prime}(0)=-\frac{1}{a\left(u_{-}\right)^{2}} \frac{\left(\nabla \lambda_{k} \cdot r_{k}\right)\left(u_{-}, a_{-}\right)}{\left(l_{k} \cdot\left(\partial_{a} f-g\right)\right)\left(u_{-}, a_{-}\right)}>0
$$

Proof. We have

$$
\left\{\begin{array} { l } 
{ \alpha ^ { \prime } = \gamma ( m ) b _ { 0 } ( \varphi , \alpha ) , } \\
{ \varphi ^ { \prime } = \gamma ( m ) r _ { 0 } ( \varphi , \alpha ) , }
\end{array} \quad \text { with } \quad \left\{\begin{array}{l}
\alpha(0)=a_{-}, \\
\varphi(0)=u_{-} .
\end{array}\right.\right.
$$

The standing wave satisfies

$$
\partial_{a} f \alpha^{\prime}+D_{u} f \varphi^{\prime}=g \alpha^{\prime},
$$

that we rewrite as

$$
\left(\partial_{a} f-g\right) \alpha^{\prime}+D_{u} f \varphi^{\prime}=0 .
$$

We decompose the vector $\varphi^{\prime}$ along the right-eigenvector as

$$
\varphi^{\prime}=\sum_{i=1}^{n} c_{i}(m) r_{i}(m) .
$$


Since $\varphi^{\prime}(0)=\left(1 / a\left(u_{-}\right)\right) r_{0}=\left(1 / a\left(u_{-}\right)\right) r_{k}$, we must have $c_{i}(0)=0$ for $i \neq k$ and $c_{k}(0)=1 / a\left(u_{-}\right)$. Differentiating (2.14) with respect to $m$ we obtain

$$
\left(\partial_{a a} f-\partial_{a} g\right)\left(\alpha^{\prime}\right)^{2}+\left(\partial_{a} f-g\right) \alpha^{\prime \prime}+D_{u}\left(\partial_{a} f-g\right) \varphi^{\prime} \alpha^{\prime}+\frac{\mathrm{d}}{\mathrm{d} m}\left\{D_{u} f \varphi^{\prime}\right\}=0 .
$$

We can rewrite the last term of the left-hand side using the decomposition (2.15). In fact,

$$
D_{u} f \varphi^{\prime}=D_{u} f \sum_{i=1}^{n} c_{i}(m) r_{i}(m)=\sum_{i=1}^{n} c_{i}(m) \lambda_{i}(m) r_{i}(m),
$$

and thus

$$
\frac{\mathrm{d}}{\mathrm{d} m}\left\{D_{u} f \varphi^{\prime}\right\}=\sum_{i=1}^{n} c_{i}^{\prime}(m) \lambda_{i}(m) r_{i}(m)+\sum_{i=1}^{n} c_{i}(m) \frac{\mathrm{d}}{\mathrm{d} m}\left\{\lambda_{i}(m) r_{i}(m)\right\} .
$$

At $m=0$ we have $\lambda_{k}(0)=0$ and $\alpha^{\prime}(0)=0$, hence

$$
\sum_{i=1}^{n} c_{i}^{\prime}(0) \lambda_{i}(0) r_{i}(0)=\sum_{i \neq k} c_{i}^{\prime}(0) \lambda_{i}(0) r_{i}(0)
$$

and

$$
\begin{aligned}
\left.\sum_{i=1}^{n} c_{i}(m) \frac{\mathrm{d}}{\mathrm{d} m}\left\{\lambda_{i}(m) r_{i}(m)\right\}\right|_{m=0} & =\left.\frac{1}{a\left(u_{-}\right)}\left\{\frac{1}{a\left(u_{-}\right)} \nabla \lambda_{k} \cdot r_{k}+\partial_{a} \lambda_{k} \alpha^{\prime}(0)\right\} r_{k}\right|_{\left(u_{-}, a_{-}\right)} \\
& =\left.\frac{1}{a\left(u_{-}\right)^{2}}\left(\nabla \lambda_{k} \cdot r_{k}\right) r_{k}\right|_{\left(u_{-}, a_{-}\right)} .
\end{aligned}
$$

We now evaluate (2.16) at $m=0$, and we obtain

$$
\left(\partial_{a} f-g\right) \alpha^{\prime \prime}+\sum_{i \neq k} c_{i}^{\prime} \lambda_{i} r_{i}+\frac{1}{a\left(u_{-}\right)^{2}}\left(\nabla \lambda_{k} \cdot r_{k}\right) r_{k}=0 .
$$

Finally, multiplying on the left by $l_{k}\left(u_{-}, a_{-}\right)$yields

$$
\alpha^{\prime \prime}(0)=-\left.\frac{1}{a\left(u_{-}\right)^{2}} \frac{\nabla \lambda_{k} \cdot r_{k}}{l_{k} \cdot\left(\partial_{a} f-g\right)}\right|_{\left(u_{-}, a_{-}\right)}>0,
$$

where we have used the hypothesis (1.7).

Remark 2.2. Lemma 2.1 shows that the standing wave curve that passes through $U_{-}=\left(u_{-}, a_{-}\right) \in \mathcal{T}$ touches the hyperplane $a=a_{-}$only at $U_{-}$and does not cross it. The sign assumptions in (1.6) and (1.7) imply that the curve lies above the hyperplane, and crosses any hyperplane $a=a_{1}, a_{1}>a_{-}$exactly twice in a neighborhood of $U_{-}$.

We note also that given $u_{-}, a_{-}$, a connecting state $u_{+}$can always be found for $a_{+}>a_{-}$, while for $a_{+}<a_{-}$this is true only as far as $a_{+} \geqslant \alpha\left(0 ; u_{-}, a_{-}\right)$. This means that smooth stationary flow is always possible for expanding ducts. On the contrary, for contracting ducts the change in area must not be too large.

We now describe the transformed standing curve corresponding to a given standing wave curve. By the sign assumption in (1.6), shock curves cross $\mathcal{T}$ from $\mathcal{T}^{+}$to $\mathcal{T}^{-}$. By the Rankine-Hugoniot condition

$$
s[u]=[f(u)],
$$

the 0 -speed shocks $(s=0)$ cross $\mathcal{T}$ at a constant value of $f$. 
Denote by $Z\left(m ; u_{-}, a_{-}\right)=(\varphi(m), \alpha(m))$ a given standing wave curve (here we will assume $Z\left(0 ; u_{-}, a_{-}\right)=$ $\left.\left(u_{-}, a_{-}\right) \in \mathcal{T}\right)$. For a given state $\left(u_{L}, a_{L}\right)=\left(\varphi\left(m_{L}\right), \alpha\left(m_{L}\right)\right) \in \mathcal{T}^{+},\left(m_{L}>0\right)$, on this curve, define $\bar{u}_{L}$ and $\tilde{u}_{L}$ such that the states $\left(\bar{u}_{L}, a_{L}\right)$ and $\left(\tilde{u}_{L}, a_{L}\right)$ lie on the other side of $\mathcal{T}$ at the same $a$-level and belong, respectively, to the same standing wave curve and to the same level curve of $f$ as the state $\left(a_{L}, u_{L}\right)$. That is, $\bar{u}_{L}$ and $\tilde{u}_{L}$ must satisfy

$$
\left(\bar{u}_{L}, a_{L}\right)=\left(\varphi\left(m_{R}\right), \alpha\left(m_{R}\right)\right)
$$

(for some $m_{R}<0$ ) and

$$
f\left(u_{L}, a_{L}\right)=f\left(\tilde{u}_{L}, a_{L}\right) .
$$

Definition 2.3. The transformed standing curve corresponding to a standing wave curve $Z\left(m ; u_{-}, a_{-}\right)=$ $(\varphi(m), \alpha(m))$ is the curve

$$
\left\{(u, a) \in \mathcal{T}^{-}: a=\alpha(m), f(u, a)=f(\varphi(m), \alpha(m)), m>0\right\} .
$$

Lemma 2.4. If $l_{k} \cdot D g . r_{k}<0$, then for each standing wave $Z\left(m ; u_{-}, a_{-}\right)$, the corresponding transformed standing curve lies closer to the transition surface $\mathcal{T}$ than $Z$. That is,

$$
\mu_{k}\left(\bar{u}_{L}\right)<\mu_{k}\left(\tilde{u}_{L}\right)<0 .
$$

If $l_{k} \cdot D g \cdot r_{k}>0$, then the corresponding transformed standing curve lies farer from the transition surface $\mathcal{T}$ than $Z$. That is,

$$
\mu_{k}\left(\tilde{u}_{L}\right)<\mu_{k}\left(\bar{u}_{L}\right)<0 .
$$

Note that the condition $l_{k} \cdot D g \cdot r_{k}<0$ above also arises in the analysis by Lien [27] on conservation laws with a moving source.

Proof. We denote the positive and negative branches of the standing wave curve as

$$
(\varphi(m), \alpha(m))= \begin{cases}\left(\varphi_{+}(m), \alpha_{+}(m)\right) & \text { if } m>0, \\ \left(\varphi_{-}(m), \alpha_{-}(m)\right) & \text { if } m<0 .\end{cases}
$$

Each of these branches can then be parametrized by $a$ :

$$
\begin{array}{ll}
\left(\varphi_{+}(m), \alpha_{+}(m)\right) \rightarrow \Phi_{+}(a), & a \geqslant a_{-}, \\
\left(\varphi_{-}(m), \alpha_{-}(m)\right) \rightarrow \Phi_{-}(a), & a \geqslant a_{-} .
\end{array}
$$

We compute

$$
\begin{aligned}
f\left(\bar{u}_{L}, a_{L}\right)-f\left(u_{L}, a_{L}\right) & =f\left(\varphi\left(m_{R}\right), \alpha\left(m_{R}\right)\right)-f\left(\varphi\left(m_{L}\right), \alpha\left(m_{L}\right)\right) \\
& =\int_{m_{L}}^{m_{R}}\left[D_{u} f \varphi^{\prime}+\partial_{a} f \alpha^{\prime}\right] \mathrm{d} m \\
& =\int_{m_{L}}^{m_{R}} g(\varphi(m), \alpha(m)) \alpha^{\prime}(m) \mathrm{d} m \\
& =\int_{m_{L}}^{0} g\left(\varphi_{+}(m), \alpha_{+}(m)\right) \alpha_{+}^{\prime}(m) \mathrm{d} m+\int_{0}^{m_{R}} g\left(\varphi_{-}(m), \alpha_{-}(m)\right) \alpha_{-}^{\prime}(m) \mathrm{d} m
\end{aligned}
$$




$$
\begin{aligned}
& =\int_{a_{L}}^{a_{-}} g\left(\Phi_{+}(a), a\right) \mathrm{d} a+\int_{a_{-}}^{a_{L}} g\left(\Phi_{-}(a), a\right) \mathrm{d} a \\
& =\int_{a_{-}}^{a_{L}}\left[g\left(\Phi_{-}(a), a\right)-g\left(\Phi_{+}(a), a\right)\right] \mathrm{d} a .
\end{aligned}
$$

Hence

$$
l_{k} \cdot\left(f\left(\bar{u}_{L}, a_{L}\right)-f\left(u_{L}, a_{L}\right)\right)=\int_{a_{-}}^{a_{L}} \int_{0}^{1} l_{k} \cdot D g\left(s \Phi_{-}(a)+(1-s) \Phi_{+}(a), a\right) \cdot\left(\Phi_{-}(a)-\Phi_{+}(a)\right) \mathrm{d} s \mathrm{~d} a,
$$

that is different from 0 thanks to (1.8). Hence the two curves are distinct. On the other side by (2.18) we have

$$
\begin{aligned}
l_{k} \cdot\left(f\left(\bar{u}_{L}, a_{L}\right)-f\left(u_{L}, a_{L}\right)\right) & =l_{k} \cdot\left(f\left(\bar{u}_{L}, a_{L}\right)-f\left(\tilde{u}_{L}, a_{L}\right)\right) \\
& =\int_{0}^{1} l_{k} \cdot D_{u} f\left(s \bar{u}_{L}+(1-s) \tilde{u}_{L}, a_{L}\right) \cdot\left(\bar{u}_{L}-\tilde{u}_{L}\right) \mathrm{d} s \\
& \sim \lambda_{k} l_{k} \cdot\left(\bar{u}_{L}-\tilde{u}_{L}\right),
\end{aligned}
$$

where $\lambda_{k}<0$ since $\bar{u}_{L}, \tilde{u}_{L} \in \mathcal{T}^{-}$. Identities (2.19) and (2.20) imply that

$$
\begin{aligned}
& l_{k} \cdot D g \cdot r_{k}<0 \quad \Rightarrow \quad \mu_{k}\left(\bar{u}_{L}\right)<\mu_{k}\left(\tilde{u}_{L}\right), \\
& l_{k} \cdot D g \cdot r_{k}>0 \quad \Rightarrow \quad \mu_{k}\left(\bar{u}_{L}\right)>\mu_{k}\left(\tilde{u}_{L}\right)
\end{aligned}
$$

(see Fig. 1).

In the next section will be also useful to know the mutual position of the transformed standing curve corresponding to $Z\left(m ; u_{L}, a_{L}\right)=(\varphi(m), \alpha(m))$ and the standing curve passing through $\left(\tilde{u}_{L}, a_{L}\right)$, that we will denote by $Z\left(m ; \tilde{u}_{L}, a_{L}\right)=(\tilde{\varphi}(m), \tilde{\alpha}(m))$ (see again Fig. 1). For a given state $a_{R}$, define $\tilde{u}_{L}^{\prime}$ and $u_{L}^{\prime \prime}$ such that the states $\left(\tilde{u}_{L}^{\prime}, a_{R}\right)$ and $\left(u_{L}^{\prime \prime}, a_{R}\right)$ lie in $\mathcal{T}^{-}$respectively on the standing curve $Z\left(m ; \tilde{u}_{L}, a_{L}\right)$ and on the transformed standing curve corresponding to $\left(u_{L}, a_{L}\right)$. That is, $\tilde{u}_{L}^{\prime}$ and $u_{L}^{\prime \prime}$ must satisfy

$$
\left(\tilde{u}_{L}^{\prime}, a_{R}\right)=\left(\tilde{\varphi}\left(m^{\prime}\right), \tilde{\alpha}\left(m^{\prime}\right)\right), \quad \text { for some } m^{\prime}<0,
$$

and

$$
f\left(u_{L}^{\prime \prime}, a_{R}\right)=f\left(u_{L}^{\prime}, a_{R}\right)=f\left(\varphi\left(m^{\prime \prime}\right), \alpha\left(m^{\prime \prime}\right)\right), \quad \text { for some } m^{\prime \prime}>0 .
$$
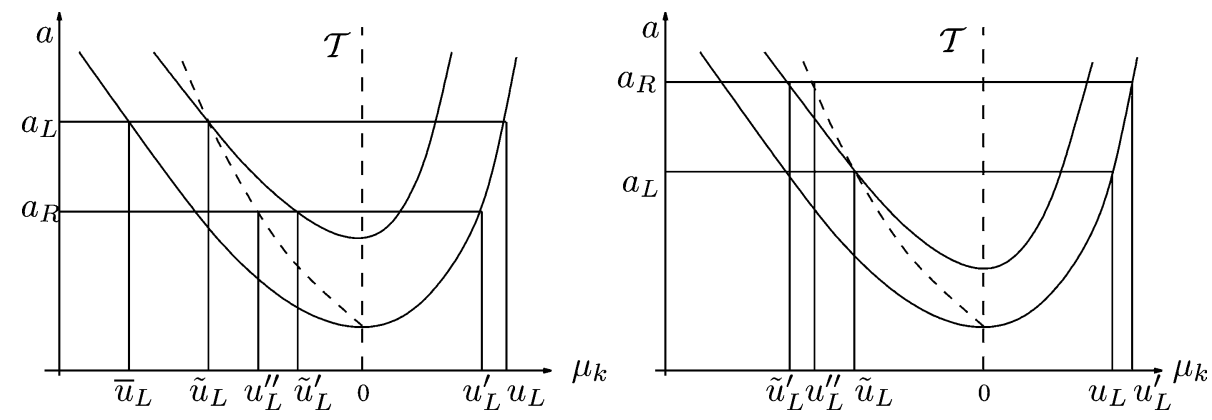

Fig. 1. The mutual position of the standing wave (continuous) and the transformed standing wave (dotted). 
Lemma 2.5. Let us assume that $l_{k} \cdot D g . r_{k}<0$. Then the following holds:

$$
\begin{aligned}
& a_{R}<a_{L} \quad \Rightarrow \quad \mu_{k}\left(u_{L}^{\prime \prime}\right)<\mu_{k}\left(\tilde{u}_{L}^{\prime}\right)<0, \\
& a_{R}>a_{L} \quad \Rightarrow \quad \mu_{k}\left(\tilde{u}_{L}^{\prime}\right)<\mu_{k}\left(u_{L}^{\prime \prime}\right)<0 .
\end{aligned}
$$

Proof. We follow closely the lines of the proof of Lemma 2.4. We compute

$$
\begin{aligned}
f\left(\tilde{u}_{L}^{\prime}, a_{R}\right)-f\left(u_{L}^{\prime \prime}, a_{R}\right)= & f\left(\tilde{\varphi}\left(m^{\prime}\right), \tilde{\alpha}\left(m^{\prime}\right)\right)-f\left(\varphi\left(m^{\prime \prime}\right), \alpha\left(m^{\prime \prime}\right)\right) \\
= & \int_{\mu_{k}\left(\tilde{u}_{L}\right)}^{m^{\prime}}\left[D_{u} f \varphi^{\prime}+\partial_{a} f \alpha^{\prime}\right] \mathrm{d} m+f\left(\tilde{u}_{L}, a_{L}\right) \\
& -\int_{\mu_{k}\left(u_{L}\right)}^{m^{\prime \prime}}\left[D_{u} f \varphi^{\prime}+\partial_{a} f \alpha^{\prime}\right] \mathrm{d} m-f\left(u_{L}, a_{L}\right) \\
= & \int_{\mu_{k}\left(\tilde{u}_{L}\right)}^{m^{\prime}} g\left(\tilde{\varphi}_{-}(m), \tilde{\alpha}_{-}(m)\right) \tilde{\alpha}_{-}^{\prime}(m) \mathrm{d} m-\int_{\mu_{k}\left(u_{L}\right)}^{m^{\prime \prime}} g\left(\varphi_{+}(m), \alpha_{+}(m)\right) \alpha_{+}^{\prime}(m) \mathrm{d} m \\
= & \int_{a_{L}}^{a_{R}} g\left(\Phi_{-}(a), a\right) \mathrm{d} a-\int_{a_{L}}^{a_{R}} g\left(\Phi_{+}(a), a\right) \mathrm{d} a \\
= & \int_{a_{L}}^{a_{R}}\left[g\left(\Phi_{-}(a), a\right)-g\left(\Phi_{+}(a), a\right)\right] \mathrm{d} a .
\end{aligned}
$$

Hence

$$
l_{k} \cdot\left(f\left(\tilde{u}_{L}^{\prime}, a_{R}\right)-f\left(u_{L}^{\prime \prime}, a_{R}\right)\right)=\int_{a_{L}}^{a_{R}} \int_{0}^{1} l_{k} \cdot D g\left(s \Phi_{-}(a)+(1-s) \Phi_{+}(a), a\right) \cdot\left(\Phi_{-}(a)-\Phi_{+}(a)\right) \mathrm{d} s \mathrm{~d} a .
$$

On the other hand we have

$$
l_{k} \cdot\left(f\left(\tilde{u}_{L}^{\prime}, a_{R}\right)-f\left(u_{L}^{\prime \prime}, a_{R}\right)\right)=\int_{0}^{1} l_{k} \cdot D_{u} f\left(s \tilde{u}_{L}^{\prime}+(1-s) u_{L}^{\prime \prime}, a_{R}\right) \cdot\left(\tilde{u}_{L}^{\prime}-u_{L}^{\prime \prime}\right) \mathrm{d} s \sim \lambda_{k} l_{k} \cdot\left(\tilde{u}_{L}^{\prime}-u_{L}^{\prime \prime}\right),
$$

where $\lambda_{k}<0$ since $\tilde{u}_{L}^{\prime}, u_{L}^{\prime \prime} \in \mathcal{T}^{-}$. Comparing identities (2.25) and (2.26) we get the conclusion.

Remark 2.6. In the case $g \equiv 0$, system (1.1) reduces to the fully conservative system

$$
\partial_{t} u+\partial_{x} f(u, a)=0,
$$

which has been studied in [18]. Note that in this case standing wave curves and 0 -speed shock curves coincide.

Remark 2.7. We consider as significant physical example the Euler equations of compressible isentropic gas flow through a nozzle 


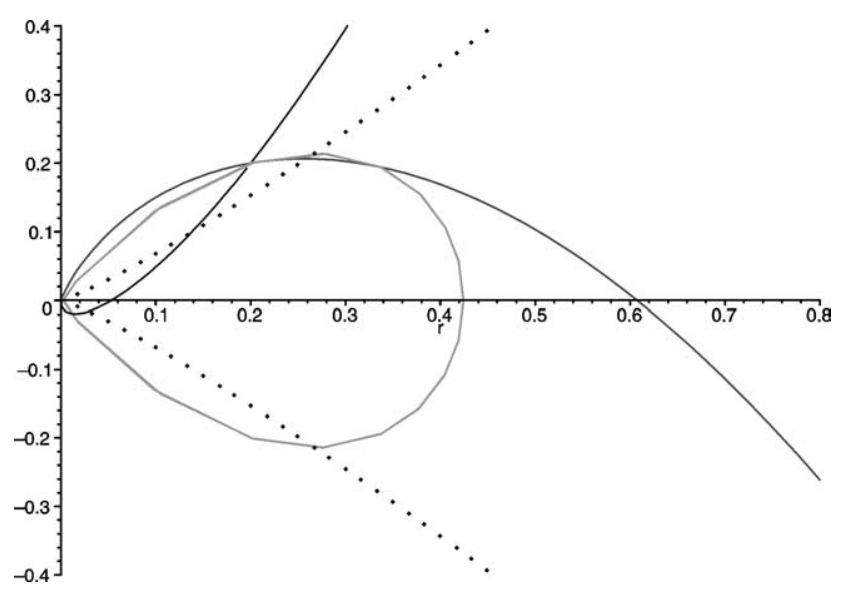

Fig. 2. Significant curves for the $2 \times 2$ Euler system.

$$
\begin{aligned}
& \partial_{t} \rho+\partial_{x} m=-\frac{m}{a} \partial_{x} a, \\
& \partial_{t} m+\partial_{x}\left(\frac{m^{2}}{\rho}+p(\rho)\right)=-\frac{m^{2}}{a \rho} \partial_{x} a,
\end{aligned}
$$

where $m$ is the momentum of the gas, $a=a(x)$ is the cross-sectional area of the duct and the pressure is given by $p(\rho)=\gamma^{-1} \rho^{\gamma}$, and $\gamma=1+2 \theta>1$ is the adiabatic constant.

The standing waves are determined by the following system of ordinary differential equations [9]:

$$
\begin{aligned}
& m_{x}=-\frac{m}{a} a_{x}, \\
& \left(\frac{m^{2}}{\rho}+p(\rho)\right)_{x}=-\frac{m^{2}}{a \rho} a_{x} .
\end{aligned}
$$

System (2.29) can be integrated, leading to the following equations which implicitly define the standing wave curve passing through a given state $\left(a_{L}, \rho_{L}, m_{L}\right)$ :

$$
\begin{aligned}
& a m=a_{L} m_{L}, \\
& \frac{m^{2}}{\rho^{2}}+\frac{1}{\theta} \rho^{2 \theta}=\frac{m_{L}^{2}}{\rho_{L}^{2}}+\frac{1}{\theta} \rho_{L}^{2 \theta} .
\end{aligned}
$$

Fig. 2 shows the projection on the $(\rho, m)$-plane of the stationary curve as well as the 1-wave curve and 2-wave curve through the point $\left(\rho_{L}, m_{L}\right)=(1 / 5,1 / 5)$, while the dotted curves $m= \pm \rho^{1+\theta}$ define the transition curves, where the eigenvalues are equal to 0 .

\section{The 0-k-curve}

As a first step toward the construction of the solution of the Riemann problem for (1.1)-(1.3), we give in this section an accurate description of the set of all right states $u=W_{0, k}\left(m ; u_{L}, a_{L}, a_{R}\right)$ associated with the level $a_{R}$ that can be reached from $\left(u_{L}, a_{L}\right)$ by a solution of the Riemann problem consisting of admissible 0 -waves and $k$-waves, only. From now on, let $a_{L}, a_{R}$ and $u_{L}$ be fixed and let us impose the following admissibility criterion on the standing waves: 
(H) A 0-wave that connects $U_{L}$ to $U_{R}$ on the same integral curve of $R_{0}$ by a contact discontinuity of speed zero is admissible if the integral curve of $R_{0}$ does not cross the transition surface $\mathcal{T}$ between $U_{L}$ and $U_{R}$.

This admissibility condition was motivated in $[18,19]$ by the fact that the total variation of $a$ in Glimm's method then does not increase. It follows from $(\mathrm{H})$ that to move to the other side of the transition surface one has to use a $k$-wave.

To construct the $0-k$-curve we will make use of the following lemma.

Lemma 3.1. There exists $\delta_{1}<\delta_{0}$ such that the following holds. Let $u_{-} \in \mathcal{B}\left(u_{*}, \delta_{1}\right)$ be given with $\mu_{k}\left(u_{-}\right)>0$ and consider the wave curve $m \mapsto \psi_{k}\left(m ; u_{-}\right)$associated to $u_{-}$. Then there exist a (unique) point $\tilde{u}_{-}$and a smooth function $\tilde{\mu}_{k} \leqslant 0$ such that

$$
\tilde{u}_{-}=v_{k}\left(\tilde{\mu}_{k}\left(u_{-}\right) ; u_{-}\right) \quad \text { and } \quad \bar{\lambda}_{k}\left(\tilde{\mu}_{k}\left(u_{-}\right) ; u_{-}\right)=0 .
$$

In particular, $\tilde{\mu}_{k}$ is a monotone decreasing function of $u_{-}$in the $r_{k}$ direction:

$\nabla \tilde{\mu}_{k} \cdot r_{k} \sim-\nabla \lambda_{k} \cdot r_{k} \quad$ near the transition manifold $\mathcal{T}$.

Proof. Existence and uniqueness of the point $\tilde{u}_{-}$is given by (1.6), which implies that the shock speed $\bar{\lambda}_{k}\left(m ; u_{-}\right)$ is strictly increasing for small $m$. We are assuming here $\tilde{u}_{-} \in \mathcal{B}\left(u_{*}, \delta_{0}\right)$, the other case being not interesting for our purpose. The second part of the statement follows from the implicit function theorem applied to the mapping $\bar{\lambda}_{k}(m ; u)$. Indeed, it is a smooth mapping of its arguments, and we have $\bar{\lambda}_{k}\left(\tilde{\mu}_{k}(u) ; u\right)=0$ by definition. Moreover from (2.11), $\partial_{m} \bar{\lambda}_{k}(m ; u)$ remains strictly positive for $m-\mu_{k}(u)$ small enough. From the definition (3.1) of $\tilde{\mu}_{k}(u)$ and (2.10) we recover

$$
\left(\tilde{\mu}_{k}(u)-\mu_{k}(u)\right)+\mathcal{O}\left(\tilde{\mu}_{k}(u)-\mu_{k}(u)\right)^{2}=-2 \mu_{k}(u),
$$

hence $\tilde{\mu}_{k}(u)-\mu_{k}(u)$ is small if $\mu_{k}(u)$ is small, that is, if $u$ is sufficiently close to the transition surface $\mathcal{T}$.

To derive (3.2) along the critical manifold we use again the implicit function theorem:

$$
\nabla \tilde{\mu}_{k} \cdot r_{k}(u)=-\frac{\nabla \bar{\lambda}_{k} \cdot r_{k}(u)}{\partial_{m} \bar{\lambda}_{k}\left(\tilde{\mu}_{k}(u) ; u\right)} .
$$

Using (2.11) and (2.12) to compute the derivatives in the right hand side of (3.3) and letting the state $u$ approach the manifold $\mathcal{T}$, we get (3.2).

The standing wave curves deserve a special treatment. As we have seen in the previous section, the standing wave through some point $\left(u_{-}, a_{-}\right)$is defined by the following ODE

$$
Z^{\prime}=\gamma(m) R_{0}(Z), \quad Z\left(\mu_{k}\left(u_{-}\right)\right)=U_{-}=\left(u_{-}, a_{-}\right)
$$

(since $R_{0} \sim R_{k}$ close to $\mathcal{T}$, we can parametrize the curve with respect to the parameter $m$ defined in (2.8)). Thanks to the regularity of $R_{0},(3.4)$ defines a curve

$$
m \mapsto Z\left(m ; U_{-}\right)=\left(\begin{array}{c}
\varphi\left(m ; u_{-}, a_{-}\right) \\
\alpha\left(m ; u_{-}, a_{-}\right)
\end{array}\right), \quad m \in(-\varepsilon, \varepsilon),
$$

for some $\varepsilon>0$, which depends smoothly upon $u_{-}, a_{-}$and $m$, and we can write the following expansions for the curve $\varphi$ and its first derivative $\partial_{m} \varphi$ :

$$
\begin{aligned}
& \varphi\left(m ; u_{-}, a_{-}\right)=u_{-}+\frac{\tilde{m}}{a\left(u_{-}\right)} r_{0}\left(u_{-}\right)+\frac{\tilde{m}^{2}}{2 a\left(u_{-}\right)^{2}}\left(D r_{0} \cdot r_{0}+b_{0} \partial_{a} r_{0}+d r_{0}\right)\left(u_{L}\right)+\mathcal{O}\left(\tilde{m}^{3}\right), \\
& \partial_{m} \varphi\left(m ; u_{-}, a_{-}\right)=\frac{1}{a\left(u_{-}\right)} r_{0}\left(u_{-}\right)+\frac{\tilde{m}}{a\left(u_{-}\right)^{2}}\left(D r_{0} \cdot r_{0}+b_{0} \partial_{a} r_{0}+d r_{0}\right)\left(u_{L}\right)+\mathcal{O}\left(\tilde{m}^{2}\right),
\end{aligned}
$$


where $d$ is some smooth function. Moreover the $R_{k}$ directional derivative with respect to the initial data is the vector $V$ which solves the linear Cauchy problem

$$
V^{\prime}=\gamma(m) D R_{0}\left(Z\left(m, U_{-}\right)\right) V, \quad V\left(\mu_{k}\left(u_{-}\right)\right)=R_{k}
$$

(see for example [17, Chapter V] for a rigorous proof). Hence, for $m-\mu_{k}\left(u_{-}\right)$sufficiently small, we have $V(m) \sim$ $R_{k}$ and, in particular,

$$
\begin{aligned}
& D_{u_{-}} \varphi\left(m ; u_{-}, a_{-}\right) . r_{k}:=\lim _{h \rightarrow 0+} \frac{\varphi\left(m ; u_{-}+h r_{k}, a_{-}\right)-\varphi\left(m ; u_{-}, a_{-}\right)}{h} \sim r_{k}, \\
& \nabla_{u_{-}} \alpha\left(m ; u_{-}, a_{-}\right) . r_{k} \sim 0 .
\end{aligned}
$$

Similarly, we can compute the derivatives w.r. to $a_{-}$. As before, they correspond to the derivative of $Z\left(m ; U_{-}\right)$w.r. to the initial data, in the direction of the vector $E_{1}=(1,0, \ldots, 0) \in \mathbb{R}^{n+1}$, that is given by the solution of

$$
V^{\prime}=\gamma(m) D R_{0}\left(Z\left(m, U_{-}\right)\right) V, \quad V\left(\mu_{k}\left(u_{-}\right)\right)=E_{1} .
$$

This means that for $m-\mu_{k}\left(u_{-}\right)$sufficiently small, we have the following approximations

$$
\begin{aligned}
& \partial_{a_{-}} \varphi\left(m ; u_{-}, a_{-}\right):=\lim _{h \rightarrow 0+} \frac{\varphi\left(m ; u_{-}, a_{-}+h\right)-\varphi\left(m ; u_{-}, a_{-}\right)}{h} \sim 0, \\
& \partial_{a_{-}} \alpha\left(m ; u_{-}, a_{-}\right) \sim 1 .
\end{aligned}
$$

We denote by $a^{T}\left(u_{-}\right)=\alpha\left(0 ; u_{-}, a_{-}\right)$the level at which the curve $Z\left(m ; U_{-}\right)$intersects the transition surface $\mathcal{T}$. In view of the admissibility criterion $(\mathbf{H})$, each fixed value $a_{+} \geqslant a^{T}\left(u_{-}\right)$uniquely defines the parameter value $\hat{\mu}\left(a_{+} ; u_{-}, a_{-}\right)$such that

$$
\alpha\left(\hat{\mu}\left(a_{+} ; u_{-}, a_{-}\right) ; u_{-}, a_{-}\right)=a_{+} .
$$

Deriving (3.12) w.r to $u_{-}$and $a_{-}$gives respectively

$$
\begin{aligned}
& D_{u_{-}} \hat{\mu}\left(a_{+} ; u_{-}, a_{-}\right) \cdot r_{k}\left(u_{-}\right) \sim 0, \\
& \partial_{m} \alpha\left(\hat{\mu}\left(a_{+} ; u_{-}, a_{-}\right) ; u_{-}, a_{-}\right) \partial_{a_{-}} \hat{\mu}\left(a_{+} ; u_{-}, a_{-}\right) \sim-1 .
\end{aligned}
$$

For $a_{L}, a_{R}$ fixed, we can thus define the map

$$
u \mapsto \varphi\left(\hat{\mu}\left(a_{R} ; u, a_{L}\right) ; u, a_{L}\right), \quad \text { for } u \in \mathcal{B}\left(u_{*}, \delta_{1}\right), a^{T}(u) \leqslant a_{R} .
$$

One should keep in mind that by (2.13), for $a_{R}<a_{L}$ a state $u$ is mapped closer to the sonic line (staying on the same side of $\mathcal{T}$ ); the opposite is true for $a_{R}>a_{L}$.

We will distinguish two main cases, depending on whether the state $U_{L}$ belongs to $\mathcal{T}^{+}$or $\mathcal{T}^{-}$.

Case 1 . We first study the case $U_{L} \in \mathcal{T}^{+}$. The analysis will be further divided into four subcases, depending on the value of $a_{R}$.

Case 1a: $a_{R} \geqslant a_{L}$. While moving along a $0-k$-curve, one has at most three possibilities:

(A) follow the standing wave curve up to level $a_{R}$, and then move along the $k$-wave with nonnegative speed;

(B) use a $k$-wave with nonpositive speed at constant level $a_{L}$ followed by a standing wave;

(C) a more complex pattern, move first along the standing wave curve up to an intermediate level $a_{M}$, jump on the other side of $\mathcal{T}$ by means of a 0 -speed $k$-shock and then use another standing wave to reach $a_{R}$.

These three cases define three different branches of the curve

$$
\widetilde{\mathcal{W}}_{k}\left(u_{L}\right):=\left\{W_{0, k}\left(m ; u_{L}, a_{L}, a_{R}\right), m \in(-\varepsilon, \varepsilon)\right\} .
$$

Case (A) defines a first branch of the curve,

$$
\tilde{\mathcal{W}}_{k}^{+}\left(u_{L}\right):=\left\{\psi_{k}\left(m ; \varphi\left(\hat{\mu}\left(a_{R} ; u_{L}, a_{L}\right) ; u_{L}, a_{L}\right)\right), m \in\left[\tilde{\mu}_{k}\left(\varphi\left(\hat{\mu}\left(a_{R} ; u_{L}, a_{L}\right) ; u_{L}, a_{L}\right)\right), \varepsilon\right)\right\},
$$




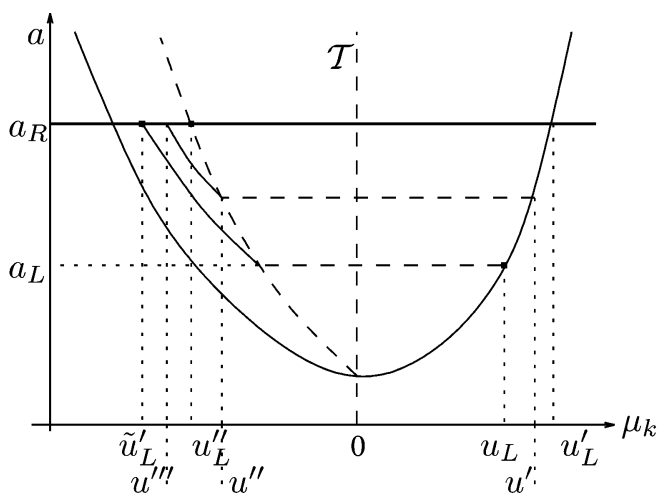

Case 1a

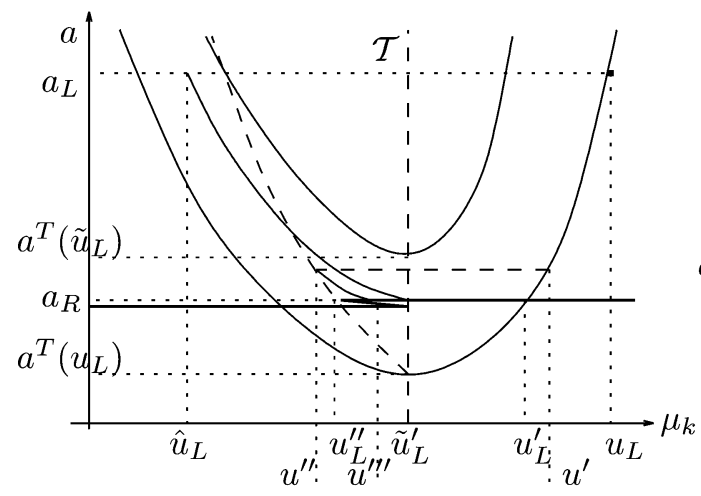

Case $1 \mathrm{c}$

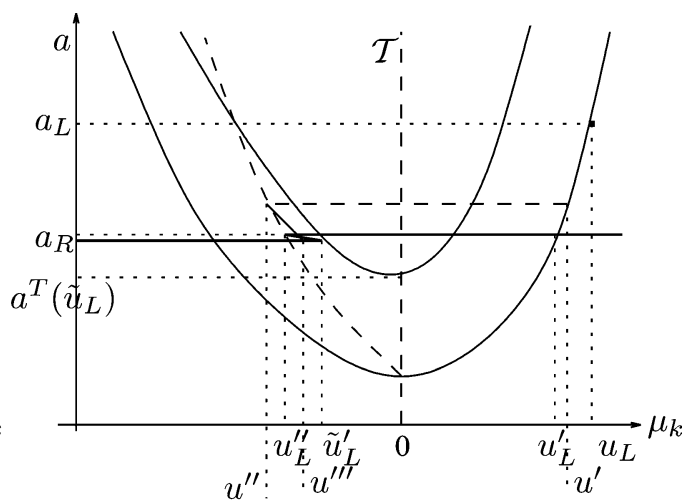

Case $1 \mathrm{~b}$

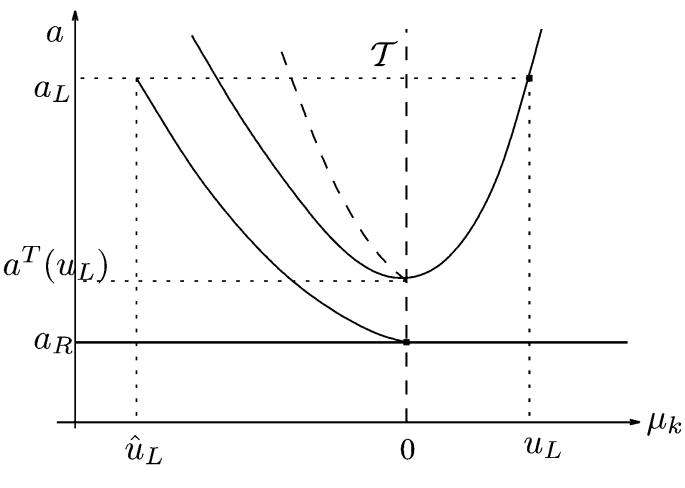

Case $1 d$

Fig. 3. Case 1.

while another branch is found following case (B)

$$
\widetilde{\mathcal{W}}_{k}^{-}\left(u_{L}\right):=\left\{\varphi\left(\hat{\mu}\left(a_{R} ; v_{k}\left(m ; u_{L}\right), a_{L}\right) ; v_{k}\left(m ; u_{L}\right), a_{L}\right), m \in\left(-\varepsilon, \tilde{\mu}_{k}\left(u_{L}\right)\right]\right\} .
$$

Finally, case (C) can be described by

$$
\begin{aligned}
\widetilde{\mathcal{W}}_{k}^{M}\left(u_{L}\right):= & \left\{\varphi\left(\hat{\mu}\left(a_{R} ; u^{\prime \prime}, \alpha\left(m ; u_{L}, a_{L}\right)\right) ; u^{\prime \prime}, \alpha\left(m ; u_{L}, a_{L}\right)\right),\right. \\
& \left.u^{\prime \prime}=v_{k}\left(\tilde{\mu}_{k}\left(\varphi\left(m ; u_{L}, a_{L}\right)\right) ; \varphi\left(m ; u_{L}, a_{L}\right)\right), \mu_{k}\left(u_{L}\right) \leqslant m \leqslant \hat{\mu}\left(a_{R} ; u_{L}, a_{L}\right)\right\} .
\end{aligned}
$$

In the following we will also set

$$
\begin{aligned}
& u^{\prime}=\varphi\left(m ; u_{L}, a_{L}\right), \quad \min \left\{a_{L}, a_{R}\right\} \leqslant \alpha\left(m ; u_{L}, a_{L}\right) \leqslant \max \left\{a_{L}, a_{R}\right\}, \\
& u^{\prime \prime \prime}=\varphi\left(\hat{\mu}\left(a_{R} ; u^{\prime \prime}, \alpha\left(m ; u_{L}, a_{L}\right)\right) ; u^{\prime \prime}, \alpha\left(m ; u_{L}, a_{L}\right)\right), \\
& u_{L}^{\prime}=\varphi\left(\hat{\mu}\left(a_{R} ; u_{L}, a_{L}\right) ; u_{L}, a_{L}\right), \\
& u_{L}^{\prime \prime}=v_{k}\left(\tilde{\mu}_{k}\left(u_{L}^{\prime}\right) ; u_{L}^{\prime}\right), \\
& \tilde{u}_{L}^{\prime}=\varphi\left(\hat{\mu}\left(a_{R} ; \tilde{u}_{L}, a_{L}\right) ; \tilde{u}_{L}, a_{L}\right)
\end{aligned}
$$

(see Fig. 3).

The setting of the following lemma is general as to cover the next cases. 
Lemma 3.2. There exists $\delta_{1}<\delta_{0}$ such that for $u_{L} \in \mathcal{B}\left(u_{*}, \delta_{1}\right)$ with $\mu_{k}\left(u_{L}\right)>0$, and $\left|a_{L}-a_{R}\right| \leqslant \delta_{1}$, the parameter $m$ is strictly monotone along each branch of the curve $\widetilde{\mathcal{W}}_{k}\left(u_{L}\right)$. More precisely

(i) $m \mapsto \lambda_{k}\left(\psi_{k}\left(m ; u_{L}^{\prime}\right)\right)$ is strictly increasing for $m \in\left[\tilde{\mu}_{k}\left(u_{L}^{\prime}\right), \varepsilon\right)$;

(ii) $m \mapsto \lambda_{k}\left(\varphi\left(\hat{\mu}\left(a_{R} ; v_{k}\left(m ; u_{L}\right), a_{L}\right) ; v_{k}\left(m ; u_{L}\right), a_{L}\right)\right)$ is strictly increasing for $m \in\left(-\varepsilon, \tilde{\mu}_{k}\left(u_{L}\right)\right]$;

(iii) if moreover $a_{R} \geqslant a^{T}\left(\tilde{u}_{L}\right)$, then $m \mapsto \lambda_{k}\left(u^{\prime \prime \prime}\right)$ is strictly increasing for $m \in\left[\mu_{k}\left(u_{L}\right), \hat{\mu}\left(a_{R} ; u_{L}, a_{L}\right)\right]$.

Proof. By definition (2.8), $m$ is strictly increasing along $\widetilde{\mathcal{W}}_{k}^{+}\left(u_{L}\right)$, and (i) is proved. To prove (ii), we use (3.7), (3.13), (2.9) and (3.8) to compute

$$
\begin{aligned}
\frac{\mathrm{d}}{\mathrm{d} m} & \varphi\left(\hat{\mu}\left(a_{R} ; v_{k}\left(m ; u_{L}\right), a_{L}\right) ; v_{k}\left(m ; u_{L}\right), a_{L}\right) \\
& =\left(\partial_{m} \varphi D_{u_{-}} \hat{\mu}+D_{u_{-}} \varphi\right) \cdot \partial_{m} v_{k}\left(m ; u_{L}\right) \\
& \sim\left(\frac{1}{a\left(u_{L}\right)} D_{u_{-}} \hat{\mu}+D_{u_{-}} \varphi\right) \cdot\left(\frac{1}{a\left(u_{L}\right)} r_{k}\left(u_{L}\right)+\mathcal{O}\left(m-\mu_{k}\left(u_{L}\right)\right)\right) \\
& \sim \frac{1}{a\left(u_{L}\right)} D_{u_{-}} \varphi \cdot r_{k}\left(u_{L}\right) \sim \frac{1}{a\left(u_{L}\right)} r_{k}\left(u_{L}\right),
\end{aligned}
$$

for $u_{L}$ sufficiently close to the transition surface, and $\left|a_{L}-a_{R}\right|$ sufficiently small.

In order to establish (iii), we need (2.9) and (3.7), which give

$$
\begin{aligned}
\frac{\mathrm{d}}{\mathrm{d} m} & v_{k}\left(\tilde{\mu}_{k}\left(\varphi\left(m ; u_{L}, a_{L}\right)\right) ; \varphi\left(m ; u_{L}, a_{L}\right)\right) \\
= & \partial_{m} v_{k}\left(\tilde{\mu}\left(u^{\prime}\right) ; u^{\prime}\right) \nabla \tilde{\mu}_{k}\left(u^{\prime}\right) \cdot \partial_{m} \varphi+D_{u} v_{k}\left(\tilde{\mu}\left(u^{\prime}\right) ; u^{\prime}\right) \cdot \partial_{m} \varphi \\
= & \left(\frac{1}{a\left(u^{\prime}\right)} r_{k}\left(u^{\prime}\right)+\mathcal{O}\left(\tilde{\mu}_{k}\left(u^{\prime}\right)-\mu_{k}\left(u^{\prime}\right)\right)\right) \nabla \tilde{\mu}_{k}\left(u^{\prime}\right) \cdot\left(\frac{1}{a\left(u_{L}\right)} r_{0}\left(u_{L}\right)+\mathcal{O}\left(m-\mu_{k}\left(u_{L}\right)\right)\right) \\
& \quad+D_{u} v_{k}\left(\tilde{\mu}\left(u^{\prime}\right) ; u^{\prime}\right) \cdot\left(\frac{1}{a\left(u_{L}\right)} r_{0}\left(u_{L}\right)+\mathcal{O}\left(m-\mu_{k}\left(u_{L}\right)\right)\right) \\
\sim & \frac{1}{a\left(u^{\prime}\right)} r_{k}\left(u^{\prime}\right) \frac{1}{a\left(u_{L}\right)} \nabla \tilde{\mu}_{k}\left(u^{\prime}\right) \cdot r_{0}\left(u_{L}\right)+\frac{1}{a\left(u_{L}\right)} D_{u} v_{k}\left(\tilde{\mu}\left(u^{\prime}\right) ; u^{\prime}\right) \cdot r_{0}\left(u_{L}\right)
\end{aligned}
$$

near $\mathcal{T}$. Moreover we compute

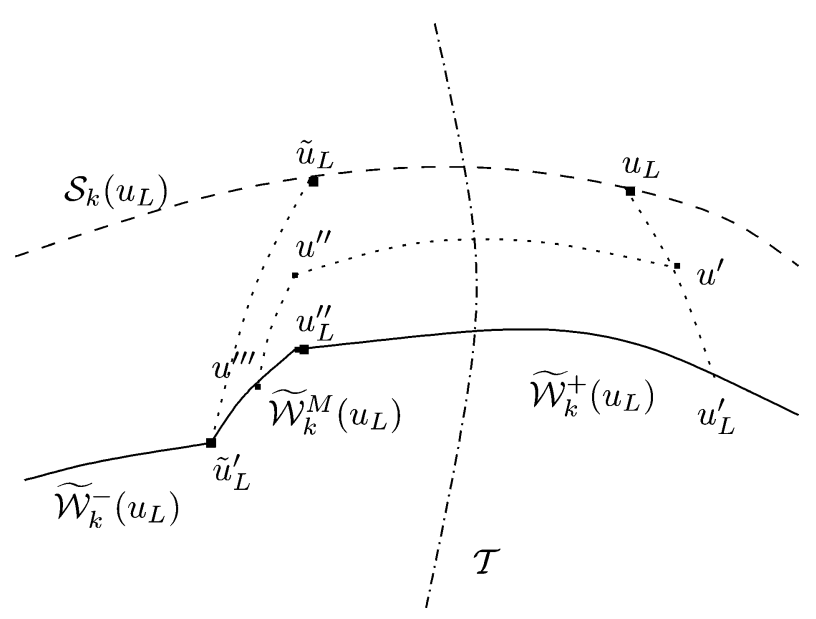

Fig. 4. The curve $\widetilde{\mathcal{W}}_{k}\left(u_{L}\right)$ for $a_{R}>a_{L}$ (case 1a). 


$$
\begin{aligned}
u^{\prime}-u_{L} & =\varphi\left(m ; u_{L}, a_{L}\right)-\varphi\left(\mu_{k}\left(u_{L}\right) ; u_{L}, a_{L}\right) \\
& =\partial_{m} \varphi\left(\mu_{k}\left(u_{L}\right) ; u_{L}, a_{L}\right)\left(m-\mu_{k}\left(u_{L}\right)\right)+\mathcal{O}\left(m-\mu_{k}\left(u_{L}\right)\right)^{2} \\
& =\frac{1}{a\left(u_{L}\right)} r_{0}\left(u_{L}\right)\left(m-\mu_{k}\left(u_{L}\right)\right)+\mathcal{O}\left(m-\mu_{k}\left(u_{L}\right)\right)^{2} .
\end{aligned}
$$

Hence we have

$$
\begin{aligned}
\tilde{\mu}\left(u^{\prime}\right) & =\tilde{\mu}\left(u_{L}\right)+\nabla \tilde{\mu}_{k}\left(u_{L}\right) \cdot\left(u^{\prime}-u_{L}\right)+\mathcal{O}\left(\left|u^{\prime}-u_{L}\right|^{2}\right) \\
& \sim \tilde{\mu}\left(u_{L}\right)+\nabla \tilde{\mu}_{k}\left(u_{L}\right) \cdot\left(\frac{1}{a\left(u_{L}\right)} r_{0}\left(u_{L}\right)\left(m-\mu_{k}\left(u_{L}\right)\right)\right) \\
& \sim \tilde{\mu}\left(u_{L}\right)+\frac{1}{a\left(u_{L}\right)} \nabla \tilde{\mu}_{k}\left(u_{L}\right) \cdot r_{0}\left(u_{L}\right)\left(m-\mu_{k}\left(u_{L}\right)\right) \\
& \sim \tilde{\mu}\left(u_{L}\right)-\frac{1}{a\left(u_{L}\right)} \nabla \lambda_{k}\left(u_{L}\right) \cdot r_{k}\left(u_{L}\right)\left(m-\mu_{k}\left(u_{L}\right)\right) \\
& \sim \tilde{\mu}\left(u_{L}\right)-\left(m-\mu_{k}\left(u_{L}\right)\right),
\end{aligned}
$$

where we have used (3.2), which also gives

$$
\begin{aligned}
\nabla \tilde{\mu}_{k}\left(u^{\prime}\right) \cdot r_{0}\left(u_{L}\right) & \sim \nabla \tilde{\mu}_{k}\left(u_{L}\right) \cdot r_{0}\left(u_{L}\right)-\nabla\left(m-\mu_{k}\left(u_{L}\right)\right) \cdot r_{0}\left(u_{L}\right) \\
& \sim \nabla \tilde{\mu}_{k}\left(u_{L}\right) \cdot r_{0}\left(u_{L}\right)+\nabla \lambda_{k}\left(u_{L}\right) \cdot r_{0}\left(u_{L}\right) \sim 0 .
\end{aligned}
$$

Using (3.16) in (3.15) we get

$$
\frac{\mathrm{d}}{\mathrm{d} m} v_{k}\left(\tilde{\mu}_{k}\left(\varphi\left(m ; u_{L}, a_{L}\right)\right) ; \varphi\left(m ; u_{L}, a_{L}\right)\right) \sim \frac{1}{a\left(u_{L}\right)} r_{0}\left(u_{L}\right)
$$

near $\mathcal{T}$. Thus, together with (3.13) and (3.14), we get the following estimate

$$
\begin{aligned}
& \frac{\mathrm{d}}{\mathrm{d} m} \varphi\left(\hat{\mu}\left(a_{R} ; v_{k}\left(\tilde{\mu}_{k}\left(u^{\prime}\right) ; u^{\prime}\right), \alpha\left(m ; u_{L}, a_{L}\right)\right) ; v_{k}\left(\tilde{\mu}_{k}\left(u^{\prime}\right) ; u^{\prime}\right), \alpha\left(m ; u_{L}, a_{L}\right)\right) \\
& \quad=\partial_{m} \varphi\left(\left(\nabla_{u_{-}} \hat{\mu}\right) \frac{\mathrm{d}}{\mathrm{d} m} v_{k}+\left(\partial_{a_{-}} \hat{\mu}\right) \partial_{m} \alpha\right)+D_{u_{-}} \varphi \cdot \frac{\mathrm{d}}{\mathrm{d} m} v_{k}+\partial_{a_{-}} \varphi \partial_{m} \alpha \\
& \quad \sim \partial_{m} \varphi\left(\frac{1}{a\left(u_{L}\right)} \nabla_{u_{-}} \hat{\mu} \cdot r_{k}\left(u_{L}\right)-C \frac{b_{0}\left(u^{\prime}, \alpha\left(m ; u_{L}, a_{L}\right)\right)}{b_{0}\left(u^{\prime \prime \prime}, a_{R}\right)}\right)+\frac{1}{a\left(u_{L}\right)} D_{u_{-}} \varphi \cdot r_{0}\left(u_{L}\right) \\
& \quad \sim-C_{1} \partial_{m} \varphi \frac{\lambda_{k}\left(u^{\prime}, \alpha\left(m ; u_{L}, a_{L}\right)\right)}{\lambda_{k}\left(u^{\prime \prime \prime}, a_{R}\right)}+\frac{1}{a\left(u_{L}\right)} r_{0}\left(u_{L}\right) \\
& \quad \sim C_{2} \frac{1}{a\left(u^{\prime \prime}\right)} r_{0}\left(u^{\prime \prime}\right)+\frac{1}{a\left(u_{L}\right)} r_{0}\left(u_{L}\right)
\end{aligned}
$$

for some smooth functions $C, C_{1}, C_{2} \sim 1$. Here we have used the fact that $\lambda_{k}\left(u^{\prime}, \alpha\left(m ; u_{L}, a_{L}\right)\right)$ and $\lambda_{k}\left(u^{\prime \prime \prime}, a_{R}\right)$ have opposite signs, since $\left(u^{\prime}, \alpha\left(m ; u_{L}, a_{L}\right)\right)$ and $\left(u^{\prime \prime \prime}, a_{R}\right)$ lie on opposite sides of $\mathcal{T}$.

It is clear that when $a_{L}=a_{R}$, the curve $\widetilde{\mathcal{W}}_{k}\left(u_{L}\right)$ coincides with $\mathcal{W}_{k}\left(u_{L}\right)$. Lemma 2.5 allows us to determine the mutual positions of the singular points $\tilde{u}_{L}^{\prime}$ and $u_{L}^{\prime \prime}$ (see Fig. 4). In this case the curve $\widetilde{\mathcal{W}}_{k}\left(u_{L}\right)$ is monotone w.r. to $m$.

Case 1b: $a^{T}\left(\tilde{u}_{L}\right) \leqslant a_{R}<a_{L}$. We still have the three branches defined by (A), (B) and (C). By Lemma 2.5 we have $\mu_{k}\left(u_{L}^{\prime \prime}\right)<\mu_{k}\left(\tilde{u}_{L}^{\prime}\right)$, hence the curve $\widetilde{\mathcal{W}}_{k}\left(u_{L}\right)$ is no more monotone w.r. to $m$, but presents a bifurcation. More precisely, $\widetilde{\mathcal{W}}_{k}^{M}\left(u_{L}\right)$ is now described by 


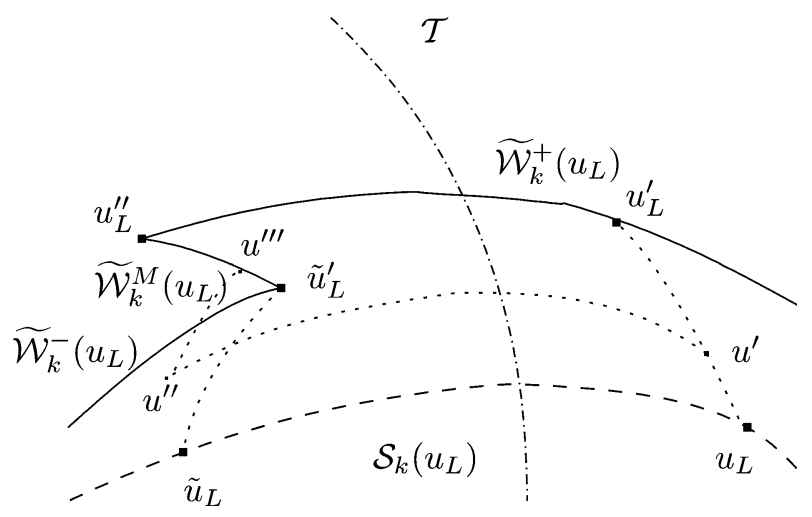

Fig. 5. The curve $\widetilde{\mathcal{W}}_{k}\left(u_{L}\right)$ for $a^{T}\left(\tilde{u}_{L}\right) \leqslant a_{R}<a_{L}$ (case $\left.1 \mathrm{~b}\right)$.

$$
\begin{aligned}
\widetilde{\mathcal{W}}_{k}^{M}\left(u_{L}\right):= & \left\{\varphi\left(\hat{\mu}\left(a_{R} ; u^{\prime \prime}, \alpha\left(m ; u_{L}, a_{L}\right)\right) ; u^{\prime \prime}, \alpha\left(m ; u_{L}, a_{L}\right)\right),\right. \\
& \left.u^{\prime \prime}=v_{k}\left(\tilde{\mu}_{k}\left(\varphi\left(m ; u_{L}, a_{L}\right)\right) ; \varphi\left(m ; u_{L}, a_{L}\right)\right), \hat{\mu}\left(a_{R} ; u_{L}, a_{L}\right) \leqslant m \leqslant \mu_{k}\left(u_{L}\right)\right\} .
\end{aligned}
$$

By Lemma 3.2, the map $m \rightarrow \lambda_{k}\left(u^{\prime \prime \prime}\right)$ is now strictly decreasing when we move from $\mu_{k}\left(u_{L}\right)$ to $\hat{\mu}\left(a_{R} ; u_{L}, a_{L}\right)$ (Fig. 5).

Case 1c: $a^{T}\left(u_{L}\right) \leqslant a_{R}<a^{T}\left(\tilde{u}_{L}\right)$. Let us define the point $\left(\hat{u}_{L}, a_{L}\right) \in \mathcal{T}^{-}$such that

$$
a^{T}\left(\hat{u}_{L}\right)=a_{R}
$$

By construction, we have

$$
\mu_{k}\left(\bar{u}_{L}\right) \leqslant \mu_{k}\left(\hat{u}_{L}\right)<\mu_{k}\left(\tilde{u}_{L}\right)=\tilde{\mu}_{k}\left(u_{L}\right)
$$

(see Lemma 2.5 and Fig. 3). Moreover we define the value $\bar{a} \in\left[a_{L}, a_{R}\right]$ such that

$$
f\left(\varphi_{+}\left(\hat{\mu}\left(\bar{a} ; u_{L}, a_{L}\right) ; u_{L}, a_{L}\right), \bar{a}\right)=f\left(\varphi_{-}\left(\hat{\mu}\left(\bar{a} ; \hat{u}_{L}, a_{L}\right) ; \hat{u}_{L}, a_{L}\right), \bar{a}\right) .
$$

That is, $\bar{a}$ is the level at which the standing wave issuing from $\left(\hat{u}_{L}, a_{L}\right)$ intersects the transformed standing wave corresponding to $Z\left(\cdot ; u_{L}, a_{L}\right)$.

Case (A) still holds, while we have cases (B) and (C) changed into

(B') use a $k$-wave with speed $\lambda_{k} \leqslant \bar{\lambda}_{k}\left(\mu_{k}\left(\hat{u}_{L}\right) ; u_{L}\right)$ at constant level $a_{L}$ followed by a standing wave;

$\left(\mathrm{C}^{\prime}\right)$ move first along the standing wave curve up to an intermediate level $a_{M}<\bar{a}$, jump on the other side of $\mathcal{T}$ by means of a 0 -speed $k$-shock and then use another standing wave to reach $a_{R}$.

Hence branches $\tilde{\mathcal{W}}_{k}^{-}\left(u_{L}\right)$ and $\tilde{\mathcal{W}}_{k}^{M}\left(u_{L}\right)$ become

$$
\begin{aligned}
\tilde{\mathcal{W}}_{k}^{-}\left(u_{L}\right):= & \left\{\varphi\left(\hat{\mu}\left(a_{R} ; v_{k}\left(m ; u_{L}\right), a_{L}\right) ; v_{k}\left(m ; u_{L}\right), a_{L}\right), m \in\left(-\varepsilon, \mu_{k}\left(\hat{u}_{L}\right)\right]\right\}, \\
\widetilde{\mathcal{W}}_{k}^{M}\left(u_{L}\right):= & \left\{\varphi\left(\hat{\mu}\left(a_{R} ; u^{\prime \prime}, \alpha\left(m ; u_{L}, a_{L}\right)\right) ; u^{\prime \prime}, \alpha\left(m ; u_{L}, a_{L}\right)\right),\right. \\
& \left.u^{\prime \prime}=v_{k}\left(\tilde{\mu}_{k}\left(\varphi\left(m ; u_{L}, a_{L}\right)\right) ; \varphi\left(m ; u_{L}, a_{L}\right)\right), \hat{\mu}\left(a_{R} ; u_{L}, a_{L}\right) \leqslant m \leqslant \hat{\mu}\left(\bar{a} ; u_{L}, a_{L}\right)\right\} .
\end{aligned}
$$

Lemma 3.2 still applies, showing that even in this case the curve $\widetilde{\mathcal{W}}_{k}\left(u_{L}\right)$ presents a bifurcation, since the map $m \rightarrow \lambda_{k}\left(u^{\prime \prime \prime}\right)$ is now strictly decreasing when we move from $\hat{\mu}\left(\bar{a} ; u_{L}, a_{L}\right)$ to $\hat{\mu}\left(a_{R} ; u_{L}, a_{L}\right)$ (Fig. 5).

Case 1d: $a_{R}<a^{T}\left(u_{L}\right)$. We take $\left(\hat{u}_{L}, a_{L}\right) \in \mathcal{T}^{-}$as defined in (3.18), except that now we have

$$
\mu_{k}\left(\hat{u}_{L}\right)<\mu_{k}\left(\bar{u}_{L}\right) \text {. }
$$

This time we can distinguish only two paths: 
(B") use a $k$-wave with speed $\lambda_{k} \leqslant \bar{\lambda}_{k}\left(\mu_{k}\left(\hat{u}_{L}\right) ; u_{L}\right)$ at constant level $a_{L}$ followed by a standing wave;

$\left(\mathrm{C}^{\prime \prime}\right)$ use a shock of speed $\lambda_{k}=\bar{\lambda}_{k}\left(\mu_{k}\left(\hat{u}_{L}\right) ; u_{L}\right)$ at level $a_{L}$, then move along the standing wave curve up to $\left(\varphi\left(\hat{\mu}\left(a_{R} ; \hat{u}_{L}, a_{L}\right) ; \hat{u}_{L}, a_{L}\right), a_{R}\right) \in \mathcal{T}$, and finally follow the rarefaction curve $w_{k}\left(m ; \varphi\left(\hat{\mu}\left(a_{R} ; \hat{u}_{L}, a_{L}\right) ; \hat{u}_{L}\right.\right.$, $\left.a_{L}\right)$ ) with positive speed at level $a_{R}$.

These define respectively the following two branches for $\widetilde{\mathcal{W}}_{k}\left(u_{L}\right)$

$$
\begin{aligned}
& \widetilde{\mathcal{W}}_{k}^{-}\left(u_{L}\right):=\left\{\varphi\left(\hat{\mu}\left(a_{R} ; v_{k}\left(m ; u_{L}\right), a_{L}\right) ; v_{k}\left(m ; u_{L}\right), a_{L}\right), m \in\left(-\varepsilon, \mu_{k}\left(\hat{u}_{L}\right)\right]\right\}, \\
& \widetilde{\mathcal{W}}_{k}^{+}\left(u_{L}\right):=\left\{w_{k}\left(m ; \varphi\left(\hat{\mu}\left(a_{R} ; \hat{u}_{L}, a_{L}\right) ; \hat{u}_{L}, a_{L}\right)\right), m \in[0, \varepsilon)\right\},
\end{aligned}
$$

which are monotone increasing w.r. to $m$ as in Lemma 3.2.

Case 2. We now study the case $U_{L} \in \mathcal{T}^{-}$. The main difference in the construction of the $0-k$-waves is that in this case we cannot "jump" on the other side of $\mathcal{T}$ by means of a $k$-shock. The analysis will be divided into three subcases, depending on the value of $a_{R}$.

Case 2a: $a_{R} \geqslant a_{L}$. We can use the following three paths:

(A) use a $k$-rarefaction with nonpositive speed at constant level $a_{L}$ up to $\left(w_{k}\left(0 ; u_{L}\right), a_{L}\right) \in \mathcal{T}$, followed by the positive branch of the standing wave up to level $a_{R}$, and finally move along a $k$-wave with nonnegative speed at constant level $a_{R}$;

(B) use a $k$-wave with nonpositive speed at constant level $a_{L}$ followed by a standing wave;

(C) move first along the $k$-rarefaction with nonpositive speed up to the point $\left(w_{k}\left(0 ; u_{L}\right), a_{L}\right) \in \mathcal{T}$, then enter $\mathcal{T}^{+}$ following the positive branch of the standing wave curve up to an intermediate level $a_{M}$, jump on the other side of $\mathcal{T}$ by means of a 0 -speed $k$-shock and then use another standing wave to reach $a_{R}$.

These three cases define respectively

$$
\begin{aligned}
& \tilde{\mathcal{W}}_{k}^{+}\left(u_{L}\right):=\left\{\psi_{k}\left(m ; \varphi_{+}\left(\hat{\mu}\left(a_{R} ; w_{k}\left(0 ; u_{L}\right), a_{L}\right) ; w_{k}\left(0 ; u_{L}\right), a_{L}\right)\right),\right. \\
&\left.m \in\left[\tilde{\mu}_{k}\left(\varphi_{+}\left(\hat{\mu}\left(a_{R} ; w_{k}\left(0 ; u_{L}\right), a_{L}\right) ; w_{k}\left(0 ; u_{L}\right), a_{L}\right)\right), \varepsilon\right)\right\}, \\
& \widetilde{\mathcal{W}}_{k}^{-}\left(u_{L}\right):=\left\{\varphi\left(\hat{\mu}\left(a_{R} ; \psi_{k}\left(m ; u_{L}\right), a_{L}\right) ; \psi_{k}\left(m ; u_{L}\right), a_{L}\right), m \in(-\varepsilon, 0]\right\}, \\
& \widetilde{\mathcal{W}}_{k}^{M}\left(u_{L}\right):=\left\{\varphi\left(\hat{\mu}\left(a_{R} ; u^{\prime \prime}, \alpha_{+}\left(m ; w_{k}\left(0 ; u_{L}\right), a_{L}\right)\right) ; u^{\prime \prime}, \alpha_{+}\left(m ; w_{k}\left(0 ; u_{L}\right), a_{L}\right)\right),\right. \\
& u^{\prime \prime}=v_{k}\left(\tilde{\mu}_{k}\left(\varphi_{+}\left(m ; w_{k}\left(0 ; u_{L}\right), a_{L}\right)\right) ; \varphi_{+}\left(m ; w_{k}\left(0 ; u_{L}\right), a_{L}\right)\right), \\
&\left.m \in\left[0, \hat{\mu}_{+}\left(a_{R} ; w_{k}\left(0 ; u_{L}\right), a_{L}\right)\right]\right\},
\end{aligned}
$$

where $\hat{\mu}_{+}$means that we are moving along $\left(\varphi_{+}, \alpha_{+}\right)$. As in case 1 , we will also set

$$
\begin{aligned}
& u^{\prime}=\varphi_{+}\left(m ; w_{k}\left(0 ; u_{L}\right), a_{L}\right), \quad a_{L} \leqslant \alpha_{+}\left(m ; w_{k}\left(0 ; u_{L}\right), a_{L}\right) \leqslant a_{R}, \\
& u^{\prime \prime \prime}=\varphi\left(\hat{\mu}\left(a_{R} ; u^{\prime \prime}, \alpha_{+}\left(m ; w_{k}\left(0 ; u_{L}\right), a_{L}\right)\right) ; u^{\prime \prime}, \alpha_{+}\left(m ; w_{k}\left(0 ; u_{L}\right), a_{L}\right)\right), \\
& u_{L}^{\prime}=\varphi_{+}\left(\hat{\mu}\left(a_{R} ; w_{k}\left(0 ; u_{L}\right), a_{L}\right) ; w_{k}\left(0 ; u_{L}\right), a_{L}\right), \\
& u_{L}^{\prime \prime}=v_{k}\left(\tilde{\mu}_{k}\left(u_{L}^{\prime}\right) ; u_{L}^{\prime}\right), \\
& \tilde{u}_{L}^{\prime}=\varphi_{-}\left(\hat{\mu}\left(a_{R} ; w_{k}\left(0 ; u_{L}\right), a_{L}\right) ; w_{k}\left(0 ; u_{L}\right), a_{L}\right)
\end{aligned}
$$

(see Fig. 6). The proof of the following lemma is very similar to the proof of Lemma 3.2.

Lemma 3.3. There exists $\delta_{1}<\delta_{0}$ such that for $u_{L} \in \mathcal{B}\left(u_{*}, \delta_{1}\right)$ with $\mu_{k}\left(u_{L}\right)<0$, and $\left|a_{L}-a_{R}\right| \leqslant \delta_{1}$, the parameter $m$ is strictly monotone along each branch of the curve $\tilde{\mathcal{W}}_{k}\left(u_{L}\right)$. More precisely

(i) $m \mapsto \lambda_{k}\left(\psi_{k}\left(m ; u_{L}^{\prime}\right)\right)$ is strictly increasing for $m \in\left[\tilde{\mu}_{k}\left(u_{L}^{\prime}\right), \varepsilon\right)$; 


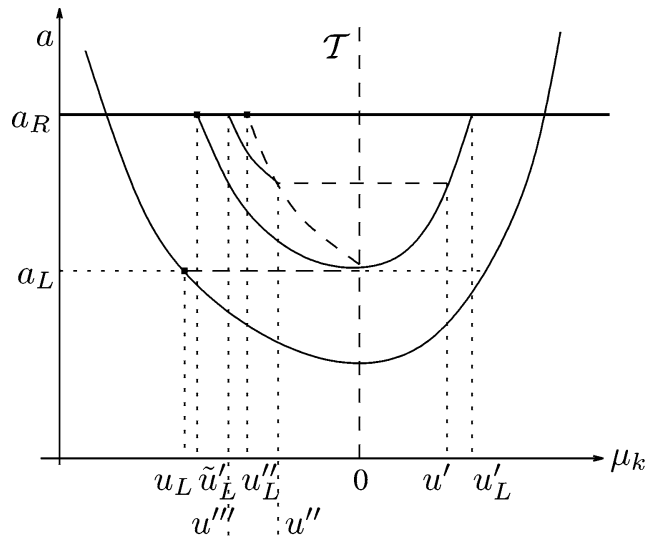

Case $2 \mathrm{a}$

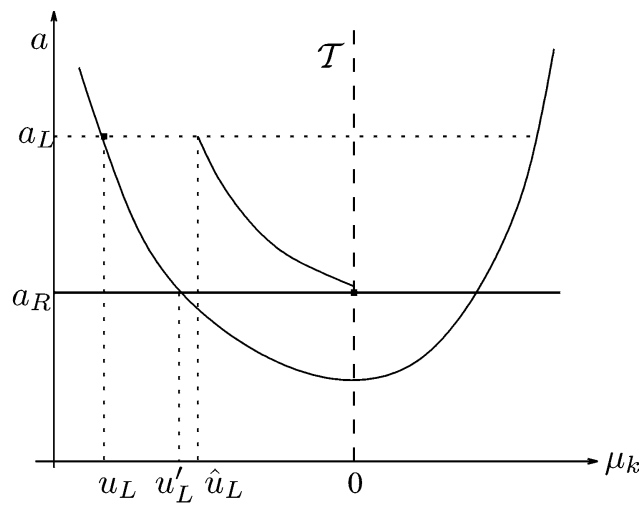

Case 2b

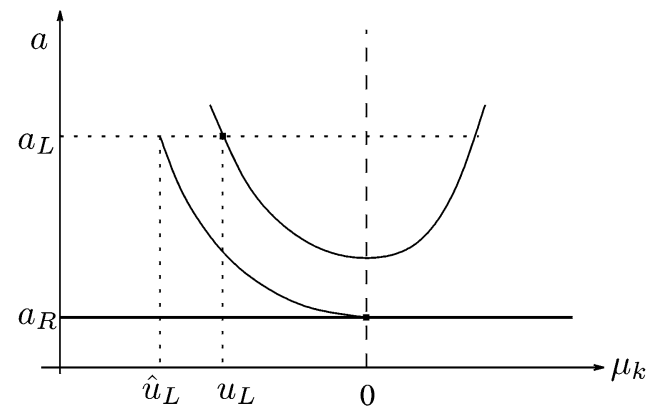

Case 2c

Fig. 6. Case 2.

(ii) $m \mapsto \lambda_{k}\left(\varphi\left(\hat{\mu}\left(a_{R} ; \psi_{k}\left(m ; u_{L}\right), a_{L}\right) ; \psi_{k}\left(m ; u_{L}\right), a_{L}\right)\right)$ is strictly increasing for $m \in(-\varepsilon, 0]$;

(iii) $m \mapsto \lambda_{k}\left(u^{\prime \prime \prime}\right)$ is strictly increasing for $m \in\left[0, \hat{\mu}\left(a_{R} ; w_{k}\left(0 ; u_{L}\right), a_{L}\right)\right]$.

Moreover, by Lemmas 2.4 and 2.5 , the singular points $\tilde{u}_{L}^{\prime}$ and $u_{L}^{\prime \prime}$ are placed so that $\mu_{k}\left(\tilde{u}_{L}^{\prime}\right) \leqslant \mu_{k}\left(u_{L}^{\prime \prime}\right)<0$. This shows that the curve $\widetilde{\mathcal{W}}_{k}\left(u_{L}\right)$ is monotone w.r. to $m$.

Case 2b: $a^{T}\left(u_{L}\right) \leqslant a_{R}<a_{L}$. In this case we have only two branches, defined by cases

$\left(\mathrm{A}^{\prime}\right)$ use a $k$-rarefaction with nonpositive speed at constant level $a_{L}$ up to $\hat{u}_{L}$ (defined as in (3.18)), then the standing wave up to level $a_{R}$, and finally move along a $k$-rarefaction with nonnegative speed at constant level $a_{R}$

$\left(\mathrm{B}^{\prime}\right)$ use a $k$-wave with nonpositive speed at constant level $a_{L}$ followed by a standing wave;

We obtain respectively the following two branches

$$
\begin{aligned}
& \tilde{\mathcal{W}}_{k}^{+}\left(u_{L}\right):=\left\{w_{k}\left(m ; \varphi\left(\hat{\mu}\left(a_{R} ; \hat{u}_{L}, a_{L}\right) ; \hat{u}_{L}, a_{L}\right)\right), m \in[0, \varepsilon)\right\}, \\
& \widetilde{\mathcal{W}}_{k}^{-}\left(u_{L}\right):=\left\{\varphi\left(\hat{\mu}\left(a_{R} ; \psi_{k}\left(m ; u_{L}\right), a_{L}\right) ; \psi_{k}\left(m ; u_{L}\right), a_{L}\right), m \in\left(-\varepsilon, \mu_{k}\left(\hat{u}_{L}\right)\right]\right\} .
\end{aligned}
$$

Again, by Lemma 3.3, the $0-k$-curve $\tilde{\mathcal{W}}_{k}\left(u_{L}\right)$ is monotone. 
Case 2c: $a_{R}<a^{T}\left(u_{L}\right)$. It is very similar to the previous case, apart from the position of $\hat{u}_{L}$, which makes ( $\left.\mathrm{A}^{\prime}\right)$ changed into

$\left(\mathrm{A}^{\prime \prime}\right)$ use a $k$-shock with nonpositive speed at constant level $a_{L}$ up to $\hat{u}_{L}$, then the standing wave up to level $a_{R}$, and finally move along a $k$-rarefaction with nonnegative speed at constant level $a_{R}$.

$\tilde{\mathcal{W}}_{k}\left(u_{L}\right)$ is still monotone.

\section{The Riemann problem}

We are now ready to solve the Riemann problem (1.1)-(1.3). First of all, since the parametrization of the $0-k$ curve exhibit jumps at the points connecting together the various branches (see the points $\tilde{u}_{L}^{\prime}, u_{L}^{\prime \prime}$ or the intersection point with $\mathcal{T}$ in Section 3), it is convenient to reparametrize the curve by choosing a global parameter $s$ so that

$$
\mu_{k}\left(W_{0, k}\left(s ; u_{L}, a_{L}, a_{R}\right)\right)=s
$$

(we set $s=m$ for the curves belonging to families $i \neq k$ ).

It is clear from the construction performed in Section 3 that the $0-k$-curve is merely Lipschitz continuous at the points $\tilde{u}_{L}^{\prime}$ and $u_{L}^{\prime \prime}$ (cases $1 \mathrm{a}, 1 \mathrm{~b}, 1 \mathrm{c}$ and $2 \mathrm{a}$ ) or at $s=0$ (cases $1 \mathrm{~d}, 2 \mathrm{~b}$ and $2 \mathrm{c}$ ), even when there is no bifurcation phenomena. So, it is necessary to rely on the implicit function theorem for Lipschitz continuous mapping [10] to obtain existence (and uniqueness) of the solution. See also [18,25].

In addition to the lack of regularity of the wave curves, we have to handle the bifurcation phenomena. So, we propose here to extend smoothly each branch of the wave curve in the $r_{k}$ direction. The corresponding curves are denoted by

$$
\begin{aligned}
& \tilde{\mathcal{W}}_{k}^{+}(u)=\left\{\widetilde{\psi}_{k}^{+}(s ; u), s \in(-\varepsilon, \varepsilon)\right\}, \\
& \widetilde{\mathcal{W}}_{k}^{-}(u)=\left\{\widetilde{\psi}_{k}^{-}(s ; u), s \in(-\varepsilon, \varepsilon)\right\}, \\
& \widetilde{\mathcal{W}}_{k}^{M}(u)=\left\{\widetilde{\psi}_{k}^{M}(s ; u), s \in(-\varepsilon, \varepsilon)\right\} .
\end{aligned}
$$

We set $\tilde{s}_{i}=s_{i}-\mu_{i}(u)$ for $i \neq k$, and $\tilde{s}_{k}=s_{k}-\mu_{i}\left(u^{\prime}\right)$, where

$$
u^{\prime}= \begin{cases}\varphi\left(\hat{\mu}\left(a_{R} ; u, a_{L}\right) ; u, a_{L}\right), & \text { if } a_{R} \geqslant a^{T}(u), \\ W_{0, k}\left(0 ; u, a_{L}, a_{R}\right), & \text { otherwise. }\end{cases}
$$

Hence each mapping

$$
\begin{aligned}
& \tilde{s}=\left(\tilde{s}_{1}, \ldots, \tilde{s}_{n}\right) \in(-\varepsilon, \varepsilon)^{n} \rightarrow \Psi^{+}(\tilde{s})=\psi_{n}\left(\tilde{s}_{n}\right) \circ \cdots \circ \widetilde{\psi}_{k}^{+}\left(\tilde{s}_{k}\right) \circ \cdots \circ \psi_{1}\left(\tilde{s}_{1}\right)\left(u_{L}\right), \\
& \tilde{s}=\left(\tilde{s}_{1}, \ldots, \tilde{s}_{n}\right) \in(-\varepsilon, \varepsilon)^{n} \rightarrow \Psi^{-}(\tilde{s})=\psi_{n}\left(\tilde{s}_{n}\right) \circ \cdots \circ \widetilde{\psi}_{k}^{-}\left(\tilde{s}_{k}\right) \circ \cdots \circ \psi_{1}\left(\tilde{s}_{1}\right)\left(u_{L}\right), \\
& \tilde{s}=\left(\tilde{s}_{1}, \ldots, \tilde{s}_{n}\right) \in(-\varepsilon, \varepsilon)^{n} \rightarrow \Psi^{M}(\tilde{s})=\psi_{n}\left(\tilde{s}_{n}\right) \circ \cdots \circ \widetilde{\psi}_{k}^{M}\left(\tilde{s}_{k}\right) \circ \cdots \circ \psi_{1}\left(\tilde{s}_{1}\right)\left(u_{L}\right)
\end{aligned}
$$

is a $\mathcal{C}^{2}$ diffeomorphism from a neighborhood of $0 \in \mathbb{R}^{n}$ onto a neighborhood of $u_{L}^{\prime}$. This follows from the implicit function theorem since the partial derivatives at $\tilde{s}=0$ are

$$
\begin{aligned}
& \partial_{i} \Psi^{ \pm, M}(0)=\alpha_{i} r_{i}\left(u_{L}, a_{L}\right), \quad i<k, \\
& \partial_{k} \Psi^{ \pm, M}(0) \sim \alpha_{k} r_{k}\left(u_{L}^{\prime}, a_{L}\right), \\
& \partial_{i} \Psi^{ \pm, M}(0) \sim \alpha_{i} r_{i}\left(u_{L}^{\prime}, a_{R}\right), \quad i>k,
\end{aligned}
$$

$\alpha_{i} \neq 0$. By the strict hyperbolicity and the continuity of $D_{u} f(u, a)$ the differentials $D \Psi^{ \pm, M}(0)$ are invertible $n \times n$ matrices. Hence there exists $\delta>0$ such that, for all $u_{L} \in \mathcal{B}\left(u_{*}, \delta_{0}\right)$, if $\left|u_{R}-u_{L}\right| \leqslant \delta$ and $\left|a_{R}-a_{L}\right| \leqslant \delta$ then 


$$
\begin{aligned}
& u_{R}=\psi_{n}\left(s_{n}^{+}\right) \circ \cdots \circ \widetilde{\psi}_{k}^{+}\left(s_{k}^{+}\right) \circ \cdots \circ \psi_{1}\left(s_{1}^{+}\right)\left(u_{L}\right), \\
& u_{R}=\psi_{n}\left(s_{n}^{-}\right) \circ \cdots \circ \widetilde{\psi}_{k}^{-}\left(s_{k}^{-}\right) \circ \cdots \circ \psi_{1}\left(s_{1}^{-}\right)\left(u_{L}\right), \\
& u_{R}=\psi_{n}\left(s_{n}^{M}\right) \circ \cdots \circ \widetilde{\psi}_{k}^{M}\left(s_{k}^{M}\right) \circ \cdots \circ \psi_{1}\left(s_{1}^{M}\right)\left(u_{L}\right),
\end{aligned}
$$

for some $s_{1}^{ \pm, M}, \ldots, s_{n}^{ \pm, M}$. Equivalently, if the points $\omega_{0}^{ \pm, M}, \ldots, \omega_{n}^{ \pm, M}$ are inductively defined by

$$
\omega_{0}=u_{L}, \quad \omega_{i}^{ \pm, M}=\psi_{i}\left(s_{i}^{ \pm, M}\right)\left(\omega_{i-1}\right),
$$

in particular

$$
\omega_{k}^{ \pm, M}=\widetilde{\psi}_{k}^{ \pm, M}\left(s_{k}^{ \pm, M}\right)\left(\omega_{k-1}\right),
$$

then $\omega_{n}^{ \pm, M}=u_{R}$. At this stage we keep into account only the solutions that are physically admissible, that is, we keep the values $s_{1}, \ldots, s_{n}$ for which the point $\omega_{k}$ belongs to one of the original branches of $\widetilde{\mathcal{W}}_{k}\left(\omega_{k-1}\right)$. Due to the transversality of each characteristic field (we recall that each branch of $\widetilde{\mathcal{W}}_{k}\left(\omega_{k-1}\right)$ is essentially parallel to $r_{k}$, and then transverse to any other curve $\left.\mathcal{W}_{i}\left(\omega_{i}\right), i \neq k\right)$, we can distinguish the following cases:

- if $\widetilde{\mathcal{W}}_{k}\left(\omega_{k-1}\right)$ has no bifurcations (cases $1 \mathrm{a}, 1 \mathrm{~d}$ and case 2 ), the solution is unique, due to the monotonicity of the parameter $s$ in the $r_{k}$ direction;

- in cases $1 \mathrm{~b}$ and $1 \mathrm{c}$ we may have up to three solutions (with one point $\omega_{k}$ on each branch of $\widetilde{\mathcal{W}}_{k}\left(\omega_{k-1}\right)$ ), which reduce to two for $s_{k}=\mu_{k}\left(\tilde{u}_{L}^{\prime}\right)$ or $s_{k}=\mu_{k}\left(u_{L}^{\prime \prime}\right)$.

Now assume that

$$
u_{R}=\psi_{n}\left(s_{n}\right) \circ \cdots \circ \widetilde{\psi}_{k}^{ \pm, M}\left(s_{k}\right) \circ \cdots \circ \psi_{1}\left(s_{1}\right)\left(u_{L}\right) .
$$

When $i \neq k$, each Riemann problem with initial data

$$
u(x, 0)=\left\{\begin{array}{ll}
\omega_{i-1}, & x<0, \\
\omega_{i}, & x>0,
\end{array} \quad a(x, 0)= \begin{cases}a_{L}, & i<k, \\
a_{R}, & i>k,\end{cases}\right.
$$

has an entropy-admissible, self-similar (that is, $u=u(x / t)$ ) solution made of two constant states separated by a contact discontinuity, a shock or a rarefaction fan.

For $i=k$, the Riemann problem with initial data

$$
(u, a)(x, 0)= \begin{cases}\left(\omega_{k-1}, a_{L}\right), & x<0, \\ \left(\omega_{k}, a_{R}\right), & x>0,\end{cases}
$$

has a self-similar solution made of two or more constant states separated by shocks, rarefactions or standing waves, that can have the same speed (equal to 0), and then be superposed. More precisely

- if $\omega_{k} \in \widetilde{\mathcal{W}}_{k}^{+}\left(\omega_{k-1}\right)$ :

Case $1 \omega_{k-1} \rightarrow \omega_{k-1}^{\prime}$ by a standing contact discontinuity, $\omega_{k-1}^{\prime} \rightarrow \omega_{k}$ by a shock or a rarefaction with nonnegative speed (one may have $\omega_{k-1}^{\prime}=\omega_{k}$ );

Case $2 \omega_{k-1} \rightarrow \omega_{k-1}^{\prime}$ by a shock or a rarefaction with nonpositive speed, $\omega_{k-1}^{\prime} \rightarrow \omega_{k-1}^{\prime \prime}$ by a standing contact discontinuity, $\omega_{k-1}^{\prime \prime} \rightarrow \omega_{k}$ by a shock or a rarefaction with nonnegative speed;

- if $\omega_{k} \in \tilde{\mathcal{W}}_{k}^{-}\left(\omega_{k-1}\right): \omega_{k-1} \rightarrow \omega_{k-1}^{\prime}$ by a shock or a rarefaction with nonpositive speed, $\omega_{k-1}^{\prime} \rightarrow \omega_{k}$ by a standing contact discontinuity;

- if $\omega_{k} \in \widetilde{\mathcal{W}}_{k}^{M}\left(\omega_{k-1}\right)$ :

Case $1 \omega_{k-1} \rightarrow \omega_{k-1}^{\prime}$ by a standing contact discontinuity, $\omega_{k-1}^{\prime} \rightarrow \omega_{k-1}^{\prime \prime}$ by a zero speed shock, $\omega_{k-1}^{\prime \prime} \rightarrow \omega_{k}$ by a standing contact discontinuity;

Case $2 \omega_{k-1} \rightarrow \omega_{k-1}^{\prime}$ by a rarefaction with nonpositive speed, $\omega_{k-1}^{\prime} \rightarrow \omega_{k-1}^{\prime \prime}$ by a standing contact discontinuity, $\omega_{k-1}^{\prime \prime} \rightarrow \omega_{k-1}^{\prime \prime \prime}$ by a zero speed shock, $\omega_{k-1}^{\prime \prime \prime} \rightarrow \omega_{k}$ by a standing contact discontinuity; 
The solution to the original problem (1.1)-(1.3) can now be constructed by piecing together the solutions of the $n$ Riemann problems (4.1)-(4.2) on different sectors of the $(x, t)$ plane. Indeed for $\tilde{s}_{1}, \ldots, \tilde{s}_{n}$ sufficiently small, and $a_{R}$ sufficiently close to $a_{L}$, the speed of each wave remains close to the corresponding eigenvalue $\lambda_{i}\left(u_{L}, a_{L}\right)$ of the matrix $D_{u} f\left(u_{L}, a_{L}\right)$. By the strict hyperbolicity and continuity properties, we can thus assume that the wave speeds remain distinct.

In conclusion, we have proved the following:

Theorem 4.1 (The Riemann solver). Suppose that, in $\mathcal{B}\left(u_{*}, \delta_{0}\right)$, the system (1.1) is strictly hyperbolic and admits only genuinely nonlinear or linearly degenerate fields. Under the assumptions (1.5)-(1.8), there exists $\delta_{1}<\delta_{0}$ such that the following holds. Given any $u_{L}, u_{R} \in \mathcal{B}\left(u_{*}, \delta_{1}\right)$, the Riemann problem (1.1)-(1.3) admits at most three self-similar solutions made up of $n+1$ constant states

$$
\omega_{0}=u_{L}, \omega_{1}, \ldots, \omega_{n}=u_{R}
$$

separated by elementary waves (shocks, rarefactions or contact discontinuities). Moreover, the states $\omega_{k-1}$ and $\omega_{k}$ are connected by at most three intermediate states $\left\{\omega_{k}^{i}\right\}_{1 \leqslant i \leqslant j, j \leqslant 3}$ separated by a standing wave and possibly shocks or rarefactions.

\section{Acknowledgements}

The first author was supported by the EC-Marie Curie Individual Fellowship \# HPMF-CT-2000-00930. The second author was supported by the Centre National de la Recherche Scientifique.

\section{References}

[1] D. Amadori, L. Gosse, G. Guerra, Global BV entropy solutions and uniqueness for hyperbolic systems of balance laws, Arch. Rational Mech. Anal. 162 (2002) 327-366.

[2] D. Amadori, L. Gosse, G. Guerra, Godunov-type approximation for a general resonant balance law with large data, J. Differential Equations 198 (2004) 233-274.

[3] N. Andrianov, G. Warnecke, The Riemann problem for the Baer-Nunziato model of two-phase flows, J. Comput. Phys., in press.

[4] N. Andrianov, G. Warnecke, On the solution to the Riemann problem for the compressible duct flow, SIAM J. Appl. Math., in press.

[5] N. Andrianov, R. Saurel, G. Warnecke, A simple method for compressible multiphase mixtures and interfaces, Int. J. Num. Meth. Fluids 41 (2003) 109-131.

[6] F. Asakura, Global solutions with a single transonic shock wave for quasilinear hyperbolic systems, submitted for publication.

[7] R. Botchorishvili, B. Perthame, A. Vasseur, Equilibrium schemes for scalar conservation laws with stiff sources, Math. of Comput. 72 (2003) 131-157.

[8] F. Bouchut, An introduction to finite volume methods for hyperbolic systems of conservation laws with source, INRIA Rocquencourt Report, 2002.

[9] G.-Q. Chen, J. Glimm, Global solutions to the compressible Euler equations with geometrical structure, Comm. Math. Phys. 180 (1996) 153-193.

[10] F.H. Clarke, Optimization and Non-Smooth Analysis, Classics in Applied Mathematics, vol. 5, Society for Industrial and Applied Mathematics (SIAM), Philadelphia, PA, 1990.

[11] R. Courant, K.O. Friedrichs, Supersonic Flow and Shock Waves, Wiley, New York, 1948.

[12] G. Dal Maso, P.G. LeFloch, F. Murat, Definition and weak stability of nonconservative products, J. Math. Pures Appl. 74 (1995) $483-548$.

[13] P. Embid, J. Goodman, J. Majda, Multiple steady states for 1-D transonic flows, SIAM J. Sci. Stat. Comput. 5 (1984) $21-41$.

[14] J. Glimm, G. Marschall, B. Plohr, A generalized Riemann problem for quasi-one-dimensional gas flows, Adv. Appl. Math. 5 (1981) 1-30.

[15] L. Gosse, A well-balanced scheme using non-conservative products designed for hyperbolic conservation laws with source-terms, Math. Model Methods Appl. Sci. 11 (2001) 339-365.

[16] J.M. Greenberg, A.Y. Leroux, A well-balanced scheme for the numerical processing of source terms in hyperbolic equations, SIAM J. Numer. Anal. 33 (1996) 1-16.

[17] P. Hartman, Ordinary Differential Equations, Wiley, New York, 1964. 
[18] E. Isaacson, B. Temple, Nonlinear resonance in systems of conservation laws, SIAM J. Appl. Math. 52 (1992) 1260-1278.

[19] E. Isaacson, B. Temple, Convergence of the $2 \times 2$ Godunov method for a general resonant nonlinear balance law, SIAM J. Appl. Math. 55 (1995) 625-640.

[20] S. Jin, X. Wen, An efficient method for computing hyperbolic systems with geometrical source terms having concentrations, J. Comp. Math. 2 (2004) 230-249.

[21] S. Jin, X. Wen, Two interface numerical methods for computing hyperbolic systems with geometrical source terms having concentrations, SIAM J. Sci. Com., in press.

[22] P.G. LeFloch, Entropy weak solutions to nonlinear hyperbolic systems in nonconservative form, Comm. Partial Differential Equations 13 (1988) 669-727.

[23] P.G. LeFloch, Shock waves for nonlinear hyperbolic systems in nonconservative form, Institute for Math. and its Appl., Minneapolis, Preprint \# 593, 1989.

[24] P.G. LeFloch, An existence and uniqueness result for two nonstrictly hyperbolic systems, in: B.L. Keyfitz, M. Shearer (Eds.), Nonlinear Evolution Equations that Change Type, in: IMA Volumes in Math. and its Appl., vol. 27, Springer-Verlag, 1990, pp. $126-138$.

[25] P.G. LeFloch, Hyperbolic Systems of Conservation Laws: The Theory of Classical and Nonclassical Shock Waves, Lectures in Mathematics, ETH Zürich, Birkhäuser, 2002.

[26] P.G. LeFloch, M.D. Thanh, The Riemann problem for fluid flows in a nozzle with discontinuous cross-section, Comm. Math. Sci. 1 (2003) 763-797.

[27] W.C. Lien, Hyperbolic conservation laws with a moving source, Comm. Pure Appl. Math. 52 (1999) 1075-1098.

[28] T.-P. Liu, Quasilinear hyperbolic systems, Comm. Math. Phys. 68 (1979) 141-172.

[29] T.-P. Liu, Nonlinear stability and instability of transonic flows through a nozzle, Comm. Math. Phys. 83 (1982) 243-260.

[30] T.-P. Liu, Nonlinear resonance for quasilinear hyperbolic equation, J. Math. Phys. 28 (1987) 2593-2602.

[31] D. Marchesin, P.J. Paes-Leme, A Riemann problem in gas dynamics with bifurcation, PUC Report MAT 02/84, 1984.

[32] D. Marchesin, B. Plohr, S. Schecter, An organizing center for wave bifurcation flow models, SIAM J. Appl. Math. 57 (1997) $1189-1215$. 